\title{
Spiders (Aranei) of the Kharaulakh Mountains and the Lena River lower reaches, northern Yakutia, Russia
}

\author{
Пауки (Aranei) Хараулахских гор и низовий р. Аена, северная \\ Якутия, Россия
}

\author{
Andrei V. Tanasevitch, Anna A. Nekhaeva \\ А.В. Танасевич, А.А. Нехаева
}

\begin{abstract}
A.N. Severtsov Institute of Ecology and Evolution, Russian Academy of Sciences, Leninsky prospekt 33, Moscow 119071, Russia. E-mail: tanasevitch@gmail.com; adrealinea@gmail.com

Институт проблем экологии и эволюции имени А.Н. Северцова РАН, Ленинский проспект 33, Москва 119071, Россия.
\end{abstract}

KEY WORDS: Arctic, typical tundra, chorology, checklist, Ust-Lensky Nature Reserve.

КЛЮЧЕВЫЕ СЛОВА: Арктика, типичные тундры, хорология, список видов, Усть-Ленский заповедник.

ABSTRACT. An analysis has been made of the two local araneofaunas: vicinities of Tyylaakh and Tiksi (northern Yakutia) situated in the subzone of typical tundra of the tundra zone. Peculiarities of spider distribution in the main zonal-landscape vegetation types were revealed. The fauna of intrazonal phytocenoses of local tundra is most rich (36 species) and specific (13\%) but characterized by a small share of purely Arctic species $(28 \%)$. Zonal groups are the second in the fauna richness (26 species) and contain the highest percentage of Arctic components (42\%). In all types of communities, the fauna basically consists of the Arctic group species, such as arcto-alpine, arcto-boreal and arcto-boreo-montane (50-58\%). Boreal species are rare and completely absent in zonal and azonal communities; polyzonal species are mainly represented in zonal and intrazonal habitats. Polytopic and petrophilous species were revealed, as well as species characteristic for certain types of vegetative aggregations. The Arctic Hilaira gertschi Holm, 1960 previously known in the Palearctic from Wrangel Island only (Chukotka Autonomous Okrug, Russia), was found for the first time in continental Siberia, in mountain tundra of the Kharaulakh Mountains. Agyneta bulavintsevi Tanasevitch, 2016 , known only from Tiksi, was for the first time recorded from Vaygach Island (Arkhangelsk Area, Russia). Sciastes hyperboreus (Kulczyński, 1908) was restored in the genus Hilaira Simon, 1884, as Hilaira hyperborea Kulczyński, 1908, comb.revalid. A complete list of spiders is given for the Lena River lower reaches, the Kharaulakh Mountains and their vicinities (99 species); a separation was drawn between species from the forest biotopes of the Lena River Valley and those of lowland tundra. Each species is characterized by its range type and the zonal component.

РЕЗЮМЕ. Проведён анализ двух локальных аранеофаун окрестностей Тыылаах и Тикси (северная
Якутия), расположенных в подзоне типичных тундр тундровой зоны. Выявлены особенности распределения пауков по основным зонально-ландшафтным типам растительности. Фауна интразональных растительных сообществ местных тундр наиболее богата (36 видов) и специфична (13\%), но характеризуется малой долей чисто арктических видов (28\%). Зональные группировки, вторые по богатству фауны (26 видов), содержат самый высокий процент арктических элементов (42\%). Во всех типах сообществ основу фауны составляют виды арктической группы, такие как, аркто-альпийские, аркто-бореальные и аркто-борео-монтанные (50-58\%). Бореальные виды представлены единично, а в зональных и азональных сообществах отсутствуют совсем; полизональные виды наиболее представлены в зональных и интразональных местообитаниях. Выявлены политопные, петрофильные, а также виды, специфичные для определённых типов растительных группировок. Арктический Hilaira gertschi Holm, 1960, ранее известный в Палеарктике лишь с острова Врангеля (Чукотский АО, Россия), впервые найден в континентальной Сибири, в горных тундрах Хараулахских гор. Agyneta bulavintsevi Tanasevitch, 2016, известный только из Тикси, впервые отмечен на о-ве Вайгач (Архангельская обл., Россия) Sciastes hyperboreus (Kulczyński, 1908) возвращён в род Hilaira Simon, 1884, Hilaira hyperborea Kulczyński, 1908, comb. revalid. Приведён полный список пауков низовий p. Лена, Хараулахских гор и их окрестностей (99 видов); разграничены виды долинных лесных биотопов р. Лена и плакорных тундр. Для каждого вида указан тип ареала и его зональная составляющая.

\section{Introduction}

The data on spiders of the Kharaulakh Mountains and lower reaches of the Lena River are few and scat- 
tered. They can be found in articles by Kulczyński [1908]; Eskov [1986, 1987, 1988] and Tanasevitch [2016]. These data were rather completely summarized by Marusik et al. [1993] in the paper on Yakutian spiders, though we had to reconsider all these data to specify the chorology of species recorded from the region.

Literature data were analyzed to separate species found in tundra landscapes from those collected in taiga (forest) habitats in the Lena River Valley. It is known that large rivers flowing northwards and crossing the tundra zone are a kind of channels for the southern faunal elements (boreal and even nemoral) moving far northwards and penetrating into non-specific natural zones (subzones) [see Chernov, Matveeva, 2002; Rybalov, 2002]. This occurs due to smoothing of pessimal conditions in valley biotopes as compared to the conditions at watersheds. An example of such penetration into the tundra is nemoral Dactylopisthes mirificus (Georgescu, 1976) found on the Barents Sea shore, in the Piesha River mouth [Tanasevitch, Koponen, 2007].

The aim of the present work was an integrative analysis of the two tundra spider faunas (Tyylaakh and Tiksi), reveal of the species distribution peculiarities in main zonal-landscape types of vegetation, as well as compilation of a corrected and complemented regional list of spiders from the Lena lower reaches, Kharaula$\mathrm{kh}$ Mountains and vicinities.

\section{Material and methods}

The present study is based on extensive spider material collected by the authors from the environs of Tyylaakh of the Ust-Lensky Nature Reserve and the vicinities of Tiksi (see Map) in July 2015. Spiders were collected by sifting moss and litter, pitfall trapping, net sweeping and hand collecting. In total, about 2000 adult spider specimens were gained and examined.

The scheme proposed by Yuri I. Chernov [1978] for classifying plant communities of the Arctic has been used for the analysis of zonal-landscape distribution of tundra spiders. Based on this classification, there are three main zonal-landscape types of plant communities in the tundra: zonal, intrazonal and azonal (for more details, see Tanasevitch \& Koponen [2007]; Tanasevitch \& Rybalov [2015]. Brief descriptions are given below for these types of vegetation in tundra in the vicinities of Tyylaakh and Tiksi. One more type is added: anthropogenic, which progressively occupies increasingly large areas in the Arctic.

Zonal plant communities (Zonal in the list below) - the plant communities that are restricted to the watershed of one zone (subzone), defining the vegetation zonal face of the landscape. In zonal communities, abiotic factors are more extreme as compared to those in intrazonal communities and therefore living conditions are more adverse. In the studied area, the zonal plant associations are represented by different variants of hummocky sedge-moss-undershrub tundra or dwarf birch/willow lichen-moss tundra, with rare and insignificant proportion of stunted undershrubs or/and creeping forms of Betula nana, Ledum and dwarf Salix spp., often with patches of bare ground. Shrubs are practically absent, except for singular willows on the Lena River bank.

Intrazonal plant communities (Intra in the list below) — the plant communities that are distributed across one or several zones (subzones); the typical examples are swamps and river valley vegetation: e.g., willow stands on floodplains, meadows, rocky and/or grassy slopes, forest stands on river banks or river terraces, etc. Commonly, parts of intrazonal communities are situated on the watershed among zonal vegetation types: these are willow shrubs in depressions, shrubby lake shores, flat-hill peatbogs with sedge fens, or sphagnum bogs in depressions, grassy slopes of small brooks, etc. The intrazonal communities are characterized by a smoother gradient of climatic factors (especially, of temperature and humidity), making living conditions there significantly less pessimal. In the studied area, the intrazonal communities are represented by variants of swampy sedge-moss or sphagnum sites in tundra, Dryas communities, lichen-moss association in rocky outcrops or rocky debris among tundra, etc.

Azonal communities (Azonal in the list below) the plant communities that are not confined to a certain zone (subzone) but distributed across many zones (subzones); in the studied area, this type is represented by specific beach plant communities on seashore, marshlands and pebble banks of the Laptev Sea, Lena and Tylakh-Yuryage rivers.

Anthropogenic communities (Antro in the list below) - it is anthropogenically changed natural vegetation, i.e. meadow formation in some tunda areas, roadsides etc., dump sites, dustbins, destroyed constructions, and so on.

It should be noted that the presented analysis of species distribution in the zonal-landscape types of vegetation is based only on the collecting efforts of the authors conducted in Tyylaakh and Tiksi in 2015, because, regretfully, the literature data on spiders of the region do not contain any ecological information.

Range types of spiders of the family Linyphiidae are given according to Tanasevitch \& Koponen [2007]; Tanasevitch \& Kamayev [2011] and Tanasevitch \& Rybalov [2010, 2015], with additions and corrections.

Abbreviations in the text. ZMMU - Zoological Museum of the Moscow State University, Moscow, Russia; MSU - Moscow State University.

\section{Region and localities}

Kharaulakh Mts (= Kharaulakh Range), elevation $200 \mathrm{~m}$ a.s.l. in the north to $1200 \mathrm{~m}$ a.s.l. in the south [Gvosdetsky, Mikhailov, 1987], are the northernmost branch of the Verkhoyansky Range in Eastern Siberia (see Map). The range vegetation is represented by various kinds of tundra, in the upper zone - by mountain tundra and goltsy. 


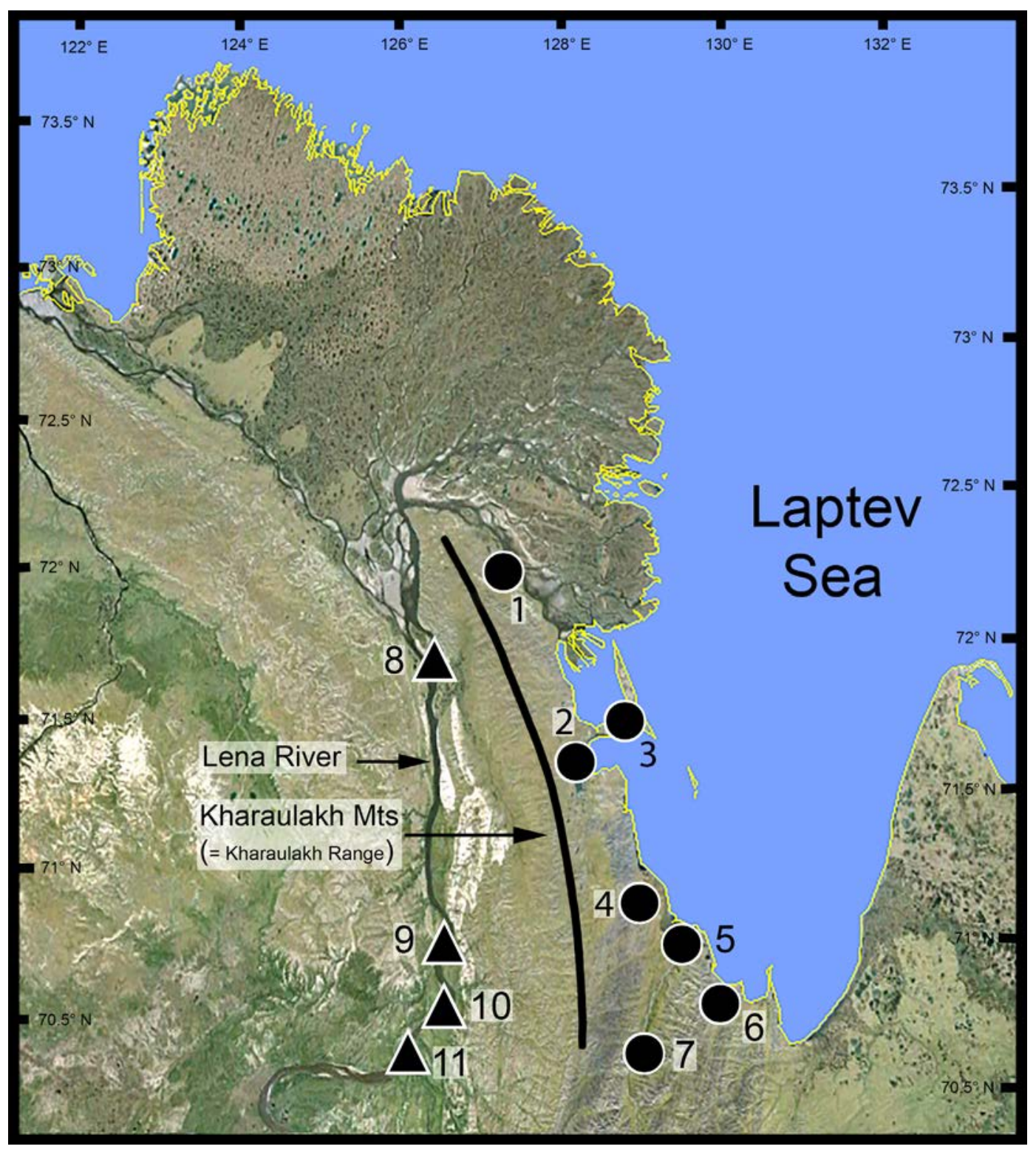

Map. Circles — localities in tundra; triangles — localities in the Lena River Valley: 1 - Tyylaakh (Ust-Lensky Nature Reserve); 2 Bulunkan Bay; Polyarka; Tiksi and Tiksi Bay; 3 - Bykovsky Peninsula; 4 - Khoptolokh River Valley; 5 - Seashore near Kharaulakh Mts; 6 - Elidep River mouth; Ketalakh-Köle Ketalakh Lake and Buor-Khaya Gulf; 7 - Antyngilkan River \& Kharaulakh River valleys; 8 - Tas-Ary \& Tit-Ary islands (Ust-Lensky Nature Reserve); 9 - Chekurovka; 10 - Bulun \& Kyusyur; 11 - Beris River.

Карта. Круги - локалитеты в тундре; треугольники - локалитеты в долине р. Лена: 1 - кордон Тыылаах (Усть-Ленский заповедник); 2 - залив Булункан, станция «Полярка», пос. Тикси и бухта Тикси; 3 - п-ов Быковский; 4 - долина р. Хоптолох; 5 берег моря у гор Хараулах; 6 - устье р. Елидеп; оз. Кеталах; Кеталах-Кёле и губа Буор-Хая; 7 - долина рек Антыгылкан и Хараулах; 8 - о-ва Тас-Ары и Тит-Ары, Усть-Ленский заповедник; 9 - Чекуровка; 10 - Булун и Кюсюр; 11 - p. Берис.

Broad valley of the Lena River lower reaches is mostly covered by small-leaved, mixed, and light forests, bogs and meadows. Light forests in depressions sometimes reach the watershed lowland but do not form vast formations there.

The authors performed spider collection in vicinities of Tyylaakh Cordon, Ust-Lensky Nature Reserve (called simply Tyylaakh below), and Tiksi (Map, No. 1 \& 2, respectively). Coordinates of collecting points and localities taken from literature are given below.
Localities in tundra landscapes (circles on the map):

1. Tyylaakh, Ust-Lensky Nature Reserve $\left(72.194709^{\circ} \mathrm{N}\right.$ $\left.128.077528^{\circ} \mathrm{E}\right)$;

2. Bulunkan Gulf Shore, Tiksi and Tiksi Bay $\left(71.622^{\circ} \mathrm{N}\right.$ $\left.128.89^{\circ} \mathrm{E}\right)$; Polyarka $\left(71.58697^{\circ} \mathrm{N} 128.85585^{\circ} \mathrm{E}\right)$;

3. Bykov Peninsula $\left(71.775^{\circ} \mathrm{N} 129.328^{\circ} \mathrm{E}\right)$;

4. Khoptolokh River (ca. $71.165^{\circ} \mathrm{N} 129.399^{\circ} \mathrm{E}$ );

5. Seashore near Kharaulakh River (ca. $71.072^{\circ} \mathrm{N}$ $\left.130.122^{\circ} \mathrm{E}\right)$

6. Elidep River mouth; Ketalakh Lake, Ketalakh-Köle and Buor-Khaya Bay (ca. $\left.70.886^{\circ} \mathrm{N} 130.415^{\circ} \mathrm{E}\right)$; 
7. Antyngilkan River and Kharaulakh River valleys $\left(70.630^{\circ} \mathrm{N} 129.589^{\circ} \mathrm{E}\right)$.

Localities in the Lena River Valley, mostly forests (triangles on the map):

8. Tas-Ary islands $\left(71.818^{\circ} \mathrm{N} 127.351^{\circ} \mathrm{E}\right)$ and Tit-Ary $\left(71.970^{\circ} \mathrm{N} 127.038^{\circ} \mathrm{E}\right)$, both belong to the Ust-Lensky Nature Reserve;

9. Chekurovka $\left(71.037^{\circ} \mathrm{N} 127.498^{\circ} \mathrm{E}\right)$;

10. Bulun $\left(70.742^{\circ} \mathrm{N} 127.401^{\circ} \mathrm{E}\right)$ and Kyusyur $\left(70.683^{\circ} \mathrm{N}\right.$ $\left.127.358^{\circ} \mathrm{E}\right)$;

11. Beris River $\left(70.520^{\circ} \mathrm{N} 127.119^{\circ} \mathrm{E}\right)$.

\section{Descriptions of plant communities}

\section{Zonal plant communities}

Hummocky tundra. Environs of Tyylaakh, site 1 (Fig. 1): $72.188050^{\circ} \mathrm{N} 128.035360^{\circ} \mathrm{E}, 9 \mathrm{~m}$ a.s.l.; site $2: 72$. $189807^{\circ} \mathrm{N} 128.075321^{\circ} \mathrm{E}, 2 \mathrm{~m}$ a.s.l. Environs of Tiksi (Fig. 2), $71.62768^{\circ} \mathrm{N} 128.90915^{\circ} \mathrm{E}, 10 \mathrm{~m}$ a.s.l. Undershrub-lichen-moss-sedge hummocky tundra with rare patches of bare ground. Sedges, mostly Eriophorum sp.; mosses: Aulacomnium turgidum, Pleurozium schreberi, Racomitrium lanuginosum, Sanionia uncinata, Polytrichum alpestre, rare spots of Sphagnum; Cladonia, Cetraria, etc.; Dactylina; Dryas, Cassiopa, Vaccinium vitis-idaea, Vaccinium uliginosum; dwarf Salix reticulata \& S. saxatilis, creeping form of Ledum and Betula nana; rare Pedicularis sp., Novosieversia glacialis, Eritrichium sp., etc.

Hummocky tundras in Tyylaakh and in Tiksi are very similar, but in the environs of Tiksi, which is $70 \mathrm{~km}$ southward of Tyylaakh, undershrubs of Vaccinium vitis-idaea and Vaccinium uliginosum are somewhat larger; Ledum and Betula nana are not of creeping form, but are small or medium sized shrubs in depressions.

Undershrub-lichen-moss tundra. Environs of Tyylaa$\underline{\mathrm{kh}}$, gentle slope at foot of the Ysy Tuoidakh-Khaya Mt. (Fig. 3), $72.18481^{\circ} \mathrm{N} 128.01111^{\circ} \mathrm{E}, 80 \mathrm{~m}$ a.s.l., occupied with undershrub-lichen-moss tundra with spots of bare ground and outcrops of stones, with Pleurozium schreberi, Aulacomnium turgidum, Racomitrium lanuginosum, Sanionia uncinata, Polytrichum alpestre; Cladonia, Cetraria; Dactylina; Dryas, Cassiopa; dwarf Salix spp. (mainly S. reticulata); beds of sedges, rare Pedicularis spp., Novosieversia glacialis, etc. Near Tylakh-Kyuel Lake (Fig. 4), $72.13364^{\circ} \mathrm{N}$ $128.01248^{\circ} \mathrm{E}, 70 \mathrm{~m}$ a.s.l., with green mosses, lichens, large spots of bare ground, sedges, Dryas, dwarf Salix spp., rare Astragalus, Eritrichium

Lichen-moss-dwarf birch tundra. Environs of Tiksi (Fig. 5), $71.61203^{\circ} \mathrm{N} 128.92281^{\circ} \mathrm{E}, 8 \mathrm{~m}$ a.s.l., with dwarf $B$. nana, Salix spp. (mainly S. reticulata); Cassiopa, Dryas, spots of Arctous, Vaccinium vitis-idaea; Aulacomnium turgidum, Pleurozium schreberi, Dicranum, Polytrichum; Ptilidium; Cladonia, Cetraria; Dactylina; rare tussocks of sedges, Pedicularis spp., Polygonum, etc.
Lichen-moss-dwarf willow tundra. Environs of Tiksi (Fig. 6), $71.61822^{\circ} \mathrm{N} 128.91891^{\circ} \mathrm{E}, 10 \mathrm{~m}$ a.s.l., with dwarf Salix spp. (mainly S. saxatilis), B. nana, Cassiopa, Dryas; Aulacomnium turgidum, Pleurozium schreberi, Polytrichum, other green mosses, spots of Sphagnum; Cladonia, Cetraria; Dactylina; tussocks of sedges; Pedicularis spp., etc.

\section{Intrazonal plant communities}

Astragalus-moss-Dryas communities. Environs of Tyylaakh, $72.185771^{\circ} \mathrm{N} 128.054779^{\circ} \mathrm{E}, 8 \mathrm{~m}$ a.s.l. Dryas tundra communities at edges of steep slopes of Tylakh-Yurge River (Fig. 7), with Astragalus spp., Pedicularis; Dryas; sedges; green mosses, etc.

Astragalus-moss-dwarf willow communities. Environs of Tyylaakh, $72.185510^{\circ} \mathrm{N} 128.061516^{\circ} \mathrm{E}, 6 \mathrm{~m}$ a.s.l. Dry channel of Tylakh-Yuryage River (Fig. 8), with dwarf Salix saxatilis, S. reticulata, Dryas; Pleurozium schreberi, Dicranum, Aulacomnium turgidum, Sanionia uncinata; sedges; Astragalus spp., Eritrichium, Pedicularis, etc.

Dwarf willow communities. Environs of Tyylaakh, bank of Tylakh-Yuryage River (Figs 9-10), 72.19381 ${ }^{\circ} \mathrm{N} 128$. $06537^{\circ} \mathrm{E}, 2 \mathrm{~m}$ a.s.l., with Salix arctica and S. reticulata; Dicranum, Pleurozium schreberi and other green mosses; Cladonia; sedges; Cassiopa, Dryas, etc.

Moss-lichen communities on rocky debris. Environs of Tiksi, high shore of Laptev Sea (Fig. 11), $71.61241^{\circ} \mathrm{N}$ $128.92384^{\circ} \mathrm{E}, 8 \mathrm{~m}$ a.s.l., with Cladonia, Cetraria; green mosses; Ledum, Dryas, Vaccinium vitis-idaea and V. uliginosum; rare Astragalus spp., etc.

Rocky outcrops in tundra. Environs of Tiksi, high shore of Laptev Sea (Fig. 12), $71.616006^{\circ} \mathrm{N} 128.918451^{\circ} \mathrm{E}$, $10 \mathrm{~m}$ a.s.1., with Cladonia, Cetraria; green mosses; Ledum, Dryas, Vaccinium vitis-idaea and V. uliginosum; rare Astragalus, etc. Near Polyarka, $71.58697^{\circ} \mathrm{N} 128.85585^{\circ} \mathrm{E}, 67 \mathrm{~m}$ a.s.l., rocky outcrops on gentle slope of hill, Cladonia, $\mathrm{Ce}$ traria; Dactylina; green mosses; Dryas, rare Astragalus, etc.

\section{Wetlands}

Moss-sedge swamps. Near Tyylaakh, site 1 (Fig. 13), $72.193762^{\circ} \mathrm{N} 128.082115^{\circ} \mathrm{E}, 4$ m a.s.l.; site $2,72.186426^{\circ} \mathrm{N}$ $128.047149^{\circ} \mathrm{E}, 12 \mathrm{~m}$ a.s.l. Environs of Tiksi: $71.61822^{\circ} \mathrm{N}$ $128.91891^{\circ} \mathrm{E}, 10 \mathrm{~m}$ a.s.l. Sedges; Dicranum, Aulacomnium palustre, Straminergon stramineum, Mnium curvatulum, Calliergon stramineum, Sphagnum; Dryas, dwarf S. reptans and $S$. reticulata, rare creeping Betula nana, etc.

Swamped bank of lake. Environs of Tyylaakh (Fig. 14), $72.19466^{\circ} \mathrm{N} 128.02940^{\circ} \mathrm{E}, 10 \mathrm{~m}$ a.s.l. Sedges, mosses: Sphagnum, Aulacomnium turgidum, Pleurozium schreberi, spots of Polytrichum alpestre; Dryas, dwarf Salix saxatilis and $S$. reticulata, etc.

\section{Azonal plant communities}

Bank of Lena River. Near Tyylaakh (Fig. 15), 72. $19700^{\circ} \mathrm{N} 128.02869^{\circ} \mathrm{E}$, floodable, pebble bank of with sedges, mosses (mostly Tomentypnum), dwarf Salix arctica, etc.

Figs. 1-10. Field photographs of the plant communities. 1, 2 - hummocky tundra, env. of Tyylaakh \& Tiksi, respectively; 3-4 undershrub-lichen-moss tundra, foot of the Ysy Tuoidakh-Khaya Mt. \& bank of Tylakh-Kyuel Lake, respectively, env. of Tyylaakh; 5 lichen-moss-dwarf birch tundra, env. of Tiksi; 6 - lichen-moss-dwarf willow tundra, env. of Tiksi; 7 - Astragalus-moss-Dryas communities, env. of Tyylaakh; 8 - Astragalus-moss-dwarf willow communities, env. of Tyylaakh; 9-10 — dwarf willow communities, env. of Tyylaakh. Photo by A. Tanasevitch $(1-3,4-10)$ and A. Nekhaeva (4).

Рис. 1-10. Фото растительных сообществ. 1, 2 - кочкарная тундра, окр. Тыылаах и Тикси, соответственно; $3-4$ - кустарничковая лишайнико-моховая тундра, подножие г. Ысы Туойдах-Хая и берег озера Тылах-Кюель, соответственно, окр. Тыылаах; 5 ерниковая лишайнико-моховая тундра, окр. Тикси; 6 - ивнячковая лишайнико-моховая тундра, окр. Тикси; 7 - астрагаломоховые дриадники, окр. Тыылаах; 8 - астрагало-моховые ивнячковые сообщества, окр. Тыылаах; 9-10 — ивнячковые сообщества, окр. Тыылаах. Фото А. Танасевича $(1-3,4-10)$ и А. Нехаевой (4). 

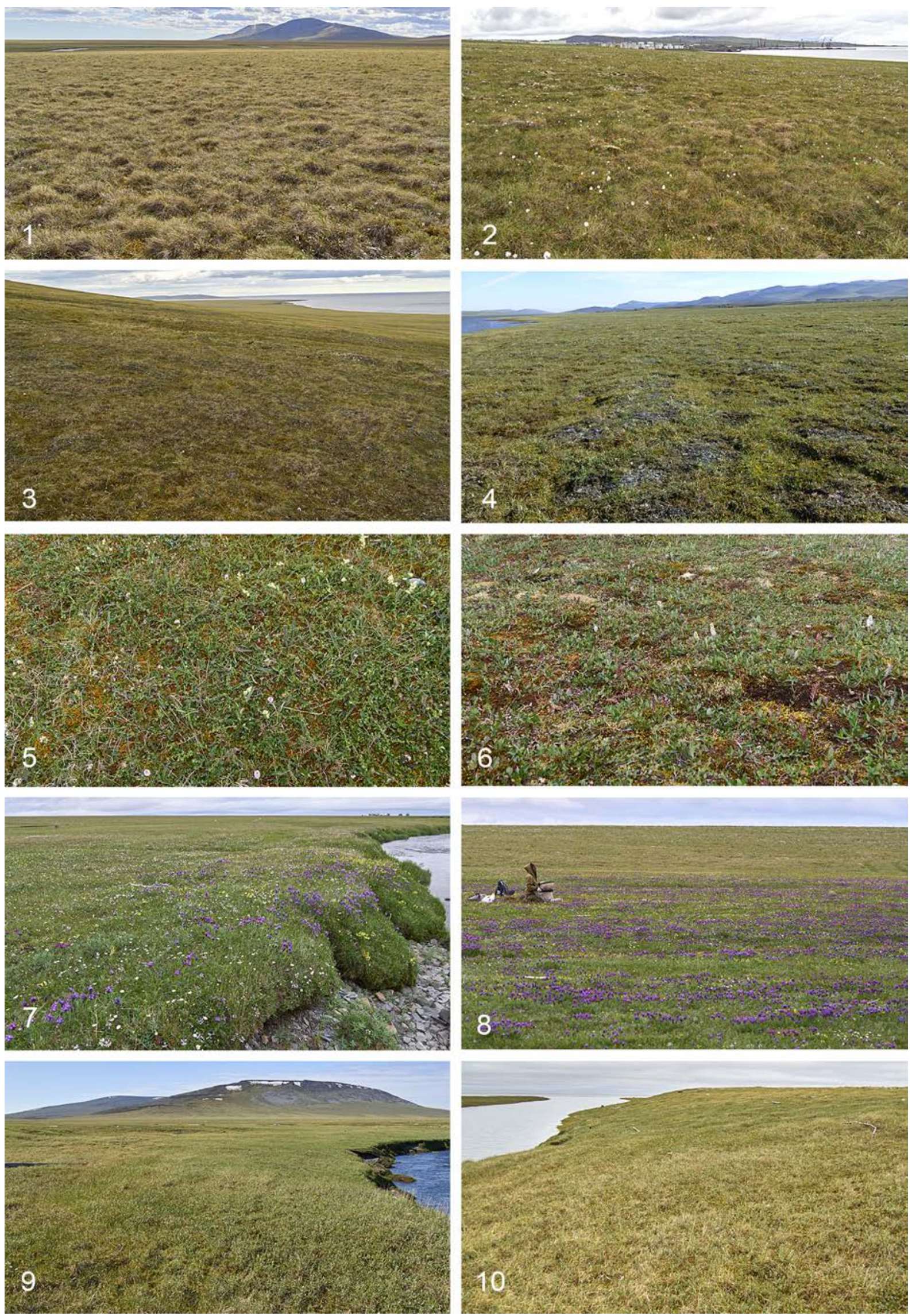

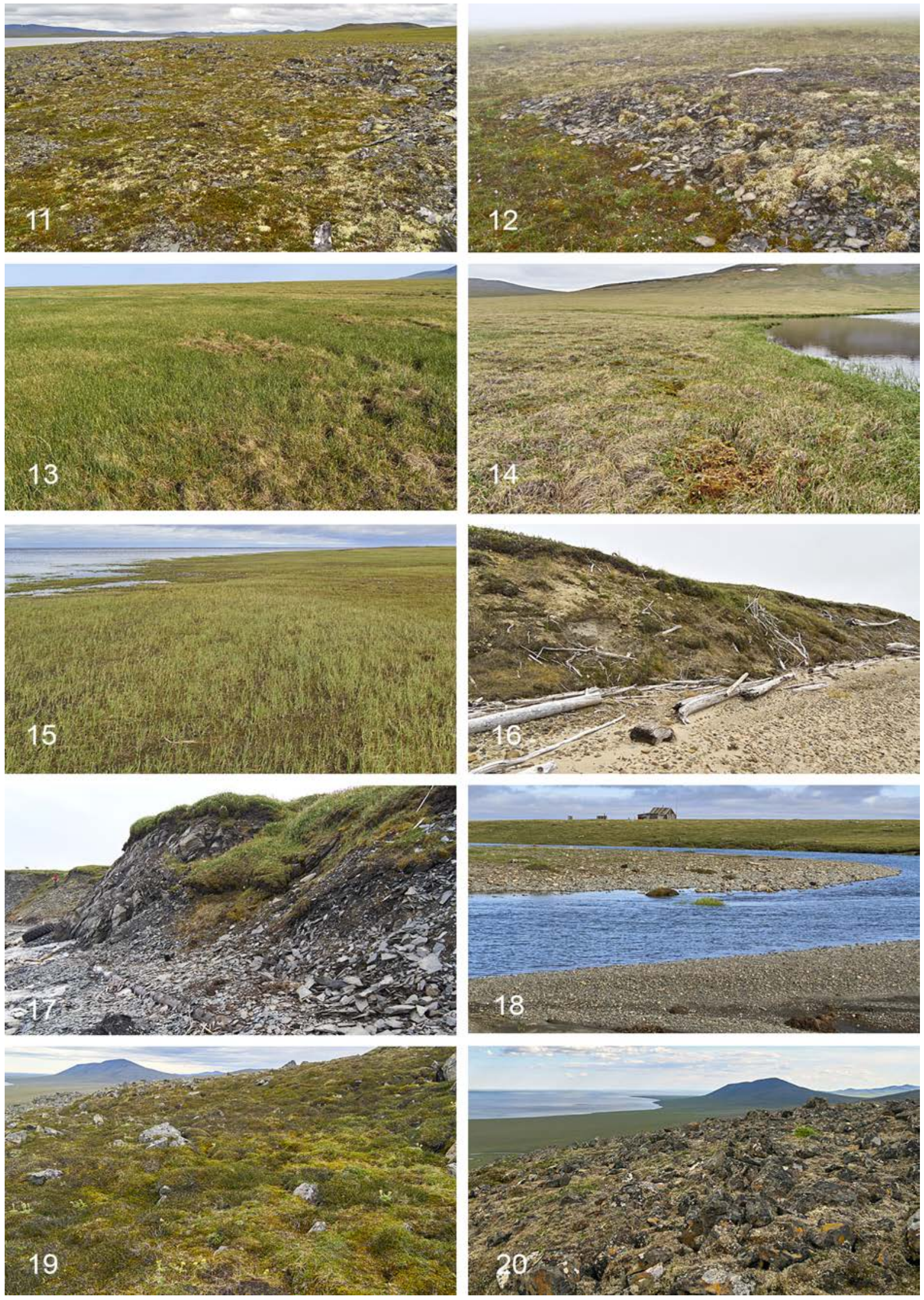
Steep slopes of Lena River banks and Laptev Sea shore. Environs of Tyylaakh, bank of Lena River (Fig. 16), $72.20533^{\circ} \mathrm{N} 127.99178^{\circ} \mathrm{E}$. Environs of Tiksi, Laptev Sea shore (Fig. 17), $71.631563^{\circ} \mathrm{N} 128.897618^{\circ} \mathrm{E}$.

Pebble bank of Tylakh-Yuryage River. Near Tyylaakh (Fig. 18), $72.193748^{\circ} \mathrm{N} 128.068624^{\circ} \mathrm{E}$.

Pebble shore of Laptev Sea. Environs of Tiksi, $71.631563^{\circ} \mathrm{N} 128.897618^{\circ} \mathrm{E}$

\section{Mountain plant communities}

Mountain tundra on rocky terrace. Environs of Tyylaakh, middle part of slope of Ysy Tuoidakh-Khaya Mt (Fig. 19), $72.18416^{\circ} \mathrm{N} 127.99687^{\circ} \mathrm{E}, 190 \mathrm{~m}$ a.s.l. Undershrub-lichen-moss tundra on rocky terrace, with Racomitrium lanuginosum, Aulacomnium turgidum, Pleurozium schreberi; sedges; Cladonia, Cetraria; Cassiopa, Dryas, dwarf Salix polaris; Saxifraga spp., rare Novosieversia glacialis, Huperzia, etc.

Mountain tundra, goltsy. Environs of Tyylaakh, summit of Ysy Tuoidakh-Khaya Mt. (Fig. 20), $72.18478^{\circ} \mathrm{N}$ $127.98512^{\circ} \mathrm{E}, 265 \mathrm{~m}$ a.s.l., undershrub-lichen and lichen mountain tundra, rocky debris on summit, with Racomitrium lanuginosum; Cladonia, Cetraria; Dactylina; dwarf Salix polaris, Cassiopa; Saxifraga spp.; rare Novosieversia glacialis, Huperzia, etc.

\section{Anthropogenic plant communities}

Grass associations in tundra. Near Tyylaakh, 72. $194709^{\circ} \mathrm{N} 128.077528^{\circ} \mathrm{E}$

Under rubbish and garbage. In Tiksi, $71.633317^{\circ} \mathrm{N}$ $128.866565^{\circ} \mathrm{E}$

\section{A list of spiders of the Kharaulakh Mts and the Lena River lower reaches}

Below are given all the species of the region known from literature and original data. Hilaira vexatrix $(\mathrm{O}$. Pickard-Cambridge, 1877) mentioned by Marusik et al. [1993] from Tit-Ary and Tas-Ary islands (Map, No. 8 ) was excluded from the list because the record was evidently based on the erroneous identification.

Families are listed in alphabetical order; the number of species is given in parentheses after the name. In the Remarks we adduce findings of the species in the studied region only.

Fam. Amaurobiidae (1)

Arctobius agelenoides (Emerton, 1919)

REMARKS. Known from the Lena River Valley: Bulun, Kyusyur \& Beris River (Map, No. 10 \& 11) [Marusik et al., 1993].
RANGE. Palaearctic-West Nearctic arcto-boreal [Nekhaeva, 2015].

Fam. Araneidae (2)

Aculepeira carbonarioides (Keyserling, 1892)

REMARKS. Known from the Lena River Valley: TitAry \& Tas-Ary islands (Map, No. 8) [Marusik et al., 1993].

RANGE. Holarctic boreal [Esyunin, Efimik, 1996; Dondale et al., 1997; Dondale et al., 2003; Paquin et al., 2010].

Aculepeira packardi (Thorell, 1875)

REMARKS. Known from Tiksi (Map, No. 2) [Marusik et al., 1993].

RANGE. Siberian-Nearctic boreo-nemoral [Dondale et al., 1997; Marusik et al., 2000; Dondale et al., 2003; Paquin et al., 2010].

Fam. Dictynidae (4)

Arctella lapponica (Holm, 1945)

REMARKS. Known from Tiksi (Map, No. 2), as well as from the Lena River Valley: Bulun, Kyusyur \& Beris River (Map, No. 10 \& 11) [Marusik et al., 1993].

RANGE. Palaearctic-West Nearctic arcto-boreal [Esyunin, Efimik, 1996; Dondale et al., 1997; Marusik et al., 2000; Paquin et al., 2010].

\section{Dictyna alaskae Chamberlin et Ivie, 1947}

REMARKS. Known from the Lena River Valley: Bulun, Kyusyur \& Beris River (Map, No. 10 \& 11) [Marusik et al., 1993].

RANGE. Holarctic boreal [Esyunin, Efimik, 1996; Dondale et al., 1997; Marusik et al., 2000; Paquin et al., 2010].

\section{Dictyna major Menge, 1869}

MATERIAL. Intra. Tiksi, rocky outcrops in tundra: 19 , among stones, 18.VII.2015; Polyarka, rocky outcrops in tundra: $1+$, among stones, 21.VII.2015.

RANGE. Holarctic polyzonal [Dondale et al., 1997; Marusik et al., 2006; Paquin et al., 2010; Marusik, Fritzén, 2011].

Emblyna borealis (O. Pickard-Cambridge, 1877)

Emblyna budarini ? - Marusik et al., 1993.

REMARKS. Known from the Lena River Valley, Tit-Ary \& Tas-Ary islands (Map, No. 8) as Emblyna budarini Marusik, 1988 [Marusik et al., 1993; Marusik, pers. com., 2016].

RANGE. East Siberian-Nearctic arctic [Marusik et al., 2006; Paquin et al., 2010].

Figs. 11-20. Field photographs of the plant communities. 11-12 - moss-lichen communities on rocky debris and rocky outcrops in tundra, env. of Tiksi; 13 - moss-sedge swamp, env. of Tyylaakh; 14 - swamped lake shore; 15 - floodable bank of the Lena River, env. of Tyylaakh; 16, 17 - steep slopes of the Lena River, env. of Tyylaakh and Laptev Sea shores near Tiksi, respectively; 18 - pebble bank of the Tylakh-Yuryage River, env. of Tyylaakh; 19 - mountain tundra on rocky terrace of Ysy Tuoidakh-Khaya Mt., env. of Tyylaakh; 20 - mountain tundra, goltsy, summit of Ysy Tuoidakh-Khaya Mt., env. of Tyylaakh. Photo by A. Tanasevitch.

Рис. 11-20. Фото растительных сообществ. 11-12 - мохово-лишайниковые сообщества среди выходов обломочных пород в тундре, окр. Тикси; 13 - мохово-осоковое болотце, окр. Тыылаах; 14 - заболоченный берег озера; 15 - заливной берег р. Лена, окр. Тыллаaх; 16-17 - береговые обрывы р. Лена, окр. Тыылаах и моря Лаптевых у Тикси, соответственно; 18 - галечный берег р. Тылах-Юряге, окр. Тыллаaх; 19 - горная тундра на террасе г. Ысы Туойдах-Хая, окр. Тыылаах; 20 — горная тундра, гольцы на вершине г. Ысы Туойдах-Хая, окр. Тыылаах. Фото А. Танасевича. 
Fam. Gnaphosidae (3)

Gnaphosa microps Holm, 1939

REMARKS. Known from the Lena River Valley: TitAry \& Tas-Ary islands (Map, No. 8) [Marusik et al., 1993].

RANGE. Holarctic boreal [Platnick, Dondale, 1992; Marusik, Koponen, 2001; Paquin et al., 2010].

Gnaphosa orites Chamberlin, 1922

REMARKS. Known from the Lena River Valley: TitAry \& Tas-Ary islands (Map, No. 8) [Marusik et al., 1993].

RANGE. Holarctic arcto-boreal [Dondale et al., 1997; Platnick, Dondale, 1992; Paquin et al., 2010; Marusik et al., 2011].

\section{Micaria alpina L. Koch, 1872}

REMARKS. Known from the Lena River Valley: TitAry \& Tas-Ary islands (Map, No. 8) [Marusik et al., 1993].

RANGE. Holarctic boreo-montane [Nekhaeva, 2015].

Fam. Hahniidae (1)

Hahnia ononidum Simon, 1875

REMARKS. Known from the Lena River Valley: Bulun, Kyusyur \& Beris River (Map, No. 10 \& 11) [Marusik et al., 1993].

RANGE. Holarctic boreo-nemoral [Nekhaeva, 2015].

Fam. Linyphiidae (66)

Agyneta affinisoides Tanasevitch, 1984

REMARKS. Known from the Lena River Valley: TitAry \& Tas-Ary islands (Map, No. 8) [Marusik et al., 1993]. RANGE. Siberian boreal.

Agyneta birulai (Kulczyński, 1908)

REMARKS. Known from the Elidep River mouth \& Buor-Khaya Bay (Map, No. 6) [Kulczyński, 1908, as Micryphantes $b$.].

RANGE. Siberian-West Nearctic arcto-boreal.

Agyneta brusnewi (Kulczyński, 1908)

MATERIAL. Intra. Tiksi, moss-lichen communities in rocky debris: $1 \overbrace{}^{7}, 1$ \%, sifting, 20.VII.2015.

REMARKS. This species was hitherto known from the Elidep River mouth and Buor-Khaya Bay (Map, No. 6) [Kulczyński, 1908, as Sintula b.].

RANGE. Siberian arctic.

\section{Agyneta bulavintsevi Tanasevitch, 2016}

REMARKS. The species was quite recently described from a male specimen from Tiksi vicinities (Map, No. 2) [Tanasevitch, 2016]. Reexamination of unidentified material from the collection of Kirill Yu. Eskov (stored at ZMMU) allowed to find several additional specimens of this species collected in Vaygach Island: $3 \sigma^{7} \sigma^{7}, 2$ 2 (ZMMU, labelled as Agyneta (Syedrula) sp. 1., det. K. Eskov), RUSSIA, Arkhangelsk Area, Vaygach Island, northern seashore, Polar Station "Bolvansky Nos", lichen-Dryas tundra, in soil sample, 4.VII.1984, leg. V. Bulavintsev. New record.

RANGE. Siberian arctic.
Agyneta decora (O. Pickard-Cambridge, 1871)

MATERIAL. Zonal. Tyylaakh, hummocky tundra: $1{ }^{7}$, pitfall traps, 13-16.VII.2015.

RANGE. Palaearctic-West Nearctic polyzonal.

Agyneta maritima (Emerton, 1919)

MATERIAL. Zonal. Tyylaakh, hummocky tundra: 8 ○ $\sigma^{\top}, 1$ o, pitfall traps, 7-16.VII.2015.

Intra. Tyylaakh, Astragalus-moss-Dryas communities: $3 \sigma^{\top 7} \sigma^{7}$, pitfall traps, 12-15.VII.2015.

RANGE. Siberian-Nearctic arcto-alpine.

Agyneta pseudosaxatilis Tanasevitch, 1984

REMARKS. Known from the Lena River Valley: TitAry \& Tas-Ary islands (Map, No. 8) [Marusik et al., 1993]. RANGE. Siberian boreal.

Arcterigone pilifrons (L. Koch, 1879)

MATERIAL. Zonal. Tyylaakh, hummocky tundra: $1 \stackrel{\circ}{\circ}$, sifting, 7.VII.2015.

Intra. Tyylaakh, moss-sedge swamp: $1 \overbrace{}^{7}, 3$ 우, sifting moss and litter, 15.VII.2015; Astragalus-moss-Dryas communities: 1 , sifting, 10.VII.2015.

REMARKS. This species was hitherto known from the Lena River Valley: Bulun, Kyusyur \& Beris River (Map, No. 10 \& 11) [Marusik et al., 1993, as Acartauchenius p.].

RANGE. Siberian-West Nearctic arctic.

Bathyphantes eumenis (L. Koch, 1879)

MATERIAL.Intra. Tiksi, rocky outcrops in tundra: $1+$, under stones, 18.VII.2015.

Mountain. Tyylaakh, mountain tundra, goltsy: 3 oq, sifting, 9.VII.2015.

REMARKS. This species was hitherto known from the Lena River Valley: Tit-Ary \& Tas-Ary islands (Map, No. 8) [Marusik et al., 1993].

RANGE. Holarctic arcto-boreal.

\section{Bathyphantes humilis (L. Koch, 1879)}

MATERIAL. Intra. Tyylaakh, dwarf willow communities: 3 $\sigma^{7} \sigma^{7}$, pitfall traps, 7-10.VII.2015; moss-sedge swamp: $1 \sigma^{7}, 12$ 웅, sifting moss and litter, 12-15.VII.2015. Tiksi, moss-sedge swamp: $2 \bigcirc^{7} \sigma^{7}, 8$, 8 , sifting moss and litter, 20.VII.2015.

Antro. Tyylaakh, grass associations: $4 \sigma^{\top} \sigma^{\top}$, pitfall traps, 1416.VII.2015.

RANGE. Siberian arcto-boreal.

Connithorax barbatus (Eskov, 1988)

REMARKS. Known from the Lena River Valley: Bulun, Kyusyur \& Beris River (Map, No. 10 \& 11) [Marusik et al., 1993].

RANGE. Siberian arcto-boreal.

Diplocephalus barbiger (Roewer, 1955)

MATERIAL. Zonal. Tiksi, lichen-moss-dwarf willow tundra: $1 \overbrace{}^{\top}, 5$ 우, sifting, 20.VII.2015.

Intra. Tyylaakh, Astragalus-moss-Dryas communities: 2 ㅇ, sweeping, 12.VII.2015; moss-sedge swamp: $2 \sigma^{\top} \sigma^{\top}, 4$ \% , sifting moss and litter, 15.VII.2015.

Mountain. Tyylaakh, mountain tundra on rocky terrace: 19 , pitfall traps, 6-15.VII.2015; mountain tundra, goltsy: $1 \sigma^{7}$, sifting, 5-9.VII.2015.

Azon. Tyylaakh, bank of Lena River: $1+$, pitfall traps, 14 16.VII.2015. 
REMARKS. This species was hitherto known from Tiksi (Map, No. 2) [Eskov, 1988, as D. barbatus].

RANGE. Siberian-Nearctic arctic.

\section{Diplocephalus mirabilis Eskov, 1988}

REMARKS. Known from the Lena River Valley: TitAry \& Tas-Ary islands, Bulun, Kyusyur \& Beris River (Map, No. 8, 10 \&11) [Marusik et al., 1993].

RANGE. Siberian boreal.

\section{Diplocephalus montanus Eskov, 1988}

REMARKS. Known from the Lena River Valley: Bulun, Kyusyur \& Beris River (Map, No. 10 \& 11) [Marusik et al., 1993].

RANGE. Siberian arcto-boreal.

\section{Diplocephalus uliginosus Eskov, 1988}

REMARKS. Known from the Lena River Valley: Bulun, Kyusyur \& Beris River (Map, No. 10 \& 11) [Marusik et al., 1993].

RANGE. Siberian boreal.

\section{Erigone arctica sibirica Kulczyński, 1908}

MATERIAL. Zonal. Tyylaakh, hummocky tundra: $5 \sigma^{7} \sigma^{7}$, pitfall traps, 7-16.VII.2015.

Intra. Tyylaakh, dwarf willow communities: $7 \sigma^{7} \sigma^{\top}, 10$ oq , pitfall traps, 7-10.VII.2015; $32 \sigma^{7} \sigma^{\top}, 24$ 우, same locality, 1016.VII.2015; Astragalus-moss-dwarf willow communities: $3 \sigma^{7} \sigma^{7}$ pitfall traps, 12-15.VII.2015; Astragalus-moss-Dryas communities: 2 + , sweeping, 12.VII.2015; 2 ㅇ, same locality, pitfall traps, 12-15.VII.2015.

Azon. Tyylaakh, bank of Lena River: $9 \sigma^{\top} \sigma^{7}, 7$ 70 , pitfall traps, 7-16.VII.2015; pebble bank of Tylakh-Yuryage River: $1 \sigma^{7}$ 2 우, under stones, 7.VII.2015; $1 \bigcirc^{7}$, same locality, 15.VII.2015. Tiksi: pebble bank of Laptev Sea: 1 , , under stones, 18.VII.2015.

Antro. Tyylaakh, grass associations: $7 \sigma^{\top} \sigma^{\top}, 11$ q, pitfall traps, 14-16.VII.2015. Tiksi, under stones and rubbish: $1 \sigma^{7}, 7$ 우, 17.VII.2015.

REMARKS. This species was hitherto known from the environs of Tiksi, Bulunkan Gulf shore (Map, No. 2) [Marusik et al., 1993].

RANGE. Siberian arcto-boreal.

\section{Erigone arcticola Chamberlin et Ivie, 1947}

REMARKS. Known from the Lena River Valley: Bulun, Kyusyur \& Beris River (Map, No. 10 \& 11) [Marusik et al., 1993].

RANGE. Siberian-West Nearctic arcto-alpine.

Erigone psychrophila Thorell, 1872

MATERIAL. Zonal. Tyylaakh, hummocky tundra: $5 \sigma^{7} \sigma^{7}, 12$ 우, pitfall traps, 7-16.VII.2015; undershrub-lichen-moss tundra: 2

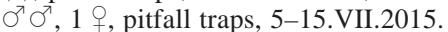

Intra. Tyylaakh, dwarf willow communities: $5 \sigma^{\top} \sigma^{\top}, 12$ 우, pitfall traps, 7-10.VII.2015; $38 \sigma^{7} \sigma^{7}, 28$ 우, same locality, 1016.VII.2015

Azon. Tyylaakh, bank of Lena River: 2 우, under stones,

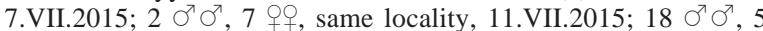
우, same locality, pitfall traps, 7-16.VII.2015.

Antro. Tyylaakh, grass associations: $18 \sigma^{\Upsilon} \sigma^{7}, 9$ 우, pitfall traps, 14-16.VII.2015

REMARKS. This species was hitherto known from the environs of Tiksi, "Kharaulakh Mountains" (without locality), Bulunkan Gulf shore and Bykovsky Peninsula (Map, No. 2 \& 3) [Kulczyński, 1908; Marusik et al., 1993].

RANGE. Holarctic arctic.

\section{Erigone tirolensis L. Koch, 1872}

MATERIAL. Intra. Tyylaakh, dwarf willow communities: 1 $\sigma^{7}$, pitfall traps, 10-13.VII.2015.

Mountain. Tyylaakh, mountain tundra on rocky terrace: $1+$ sifting, 9.VII.2015; $5 \sigma^{\top} \sigma^{7}, 1$, same locality, pitfall traps, 59.VII.2015; mountain tundra, goltsy: $15 \sigma^{\top} \sigma^{7}, 4$ 4 , pitfall traps, 6-13.VII.2015.

RANGE. Holarctic arcto-alpine.

\section{Frontella pallida Kulczyński, 1908}

REMARKS. Not recorded since the original description based on a female from the Elidep River mouth (Map, No. 6) [Kulczyński, 1908].

\section{Gibothorax tchernovi Eskov, 1989}

MATERIAL. Intra. Tyylaakh, moss-sedge swamp: $1 \sigma^{7} \& 1 \sigma^{7}$ subad., sifting moss and litter, 15.VII.2015.

Azon. Tyylaakh, bank of Lena River: $1 \sigma^{7}$, pitfall traps, 1014.VII.2015.

RANGE. Siberian arctic.

\section{Halorates holmgreni (Thorell, 1871)}

MATERIAL. Intra. Tyylaakh, dwarf willow communities: 1 ơ, pitfall traps, 13-16.VII.2015.

Tiksi, moss-lichen communities in rocky debris: $1 \sigma^{7}$, pitfall traps, 18-20.VII.2015.

Mountain. Tyylaakh, mountain tundra on rocky terrace: 3 $\sigma^{\top} \sigma^{\top}, 8$ 우, sifting, 5.VII.2015; $31 \sigma^{\top} \sigma^{\top}, 67$ 우, same locality, pitfall traps, 5-13.VII.2015; mountain tundra, goltsy: $18 \sigma^{7} \sigma^{\top}, 49$ 우, pitfall traps, 5-15.VII.2015.

Azon. Tiksi, pebble bank of Laptev Sea: $2 \sigma^{7} \sigma^{7}, 12$ 우, 25 $\sigma^{7} \sigma^{7} \&$ \& 우 subad., under stones, 18.VII.2015.

RANGE. Holarctic arcto-alpine.

Hilaira asiatica Eskov, 1987

REMARKS. Known from the Lena River Valley: Bulun, Kyusyur \& Beris River (Map, No. 10 \& 11) [Marusik et al., 1993].

RANGE. Siberian arcto-boreal.

\section{Hilaira gertschi Holm, 1960}

MATERIAL. Mountain. Tyylaakh, mountain tundra, goltsy: 1 ơ, 1 \%, sifting, 9-13.VII.2015.

REMARKS. In the Palearctic, this species was hitherto known from Wrangel Island only [Eskov, 1985]. Delta of the Lena River is the westernmost limit of its known distribution.

RANGE. Siberian-Alaskan arctic.

\section{Hilaira gibbosa Tanasevitch, 1982}

REMARKS. Known from the Lena River Valley: Bulun, Kyusyur \& Beris River (Map, No. 10 \& 11) [Marusik et al., 1993].

RANGE. Siberian-Nearctic boreal.

\section{Hilaira glacialis (Thorell, 1871)}

MATERIAL. Zonal. Tyylaakh, hummocky tundra: $1 \sigma^{7}, 7$ 우, sifting, 7.VII.2015; 1 T, 13 of, same locality, pitfall traps, 716.VII.2015; undershrub-lichen-moss tundra: 5 O+ , pitfall traps, 515.VII.2015; $2 \sigma^{7} \sigma^{7}, 3$ 우, same locality, pitfall traps, 5-9.VII.2015. Tiksi, hummocky tundra: $1 \sigma^{\top}, 10$ oq, sifting, 20.VII.2015; lichenmoss-dwarf birch tundra: 1 \%, 3 우, sifting, 20.VII.2015; 6 우, same locality, pitfall traps, 18-20.VII.2015; lichen-moss-dwarf willow tundra: $3 \sigma^{\top} \sigma^{\top}, 4$ 우, sifting, 20.VII.2015. 
Intra. Tyylaakh, Astragalus-moss-dwarf willow communities: $1 \sigma^{\nearrow}$, pitfall traps, 12-15.VII.2015; 1 \%, same locality, sifting, 15.VII.2015; moss-sedge swamp: 2 우, sifting moss and litter, $12-$ 15.VII.2015; Astragalus-moss-Dryas communities: 3 우, sifting, 10-15.VII.2015; 2 우, same locality, pitfall traps, 12-15.VII.2015.

Mountain. Tyylaakh, mountain tundra on rocky terrace: $1 \sigma^{7}$, 3 우, sifting, 5-9.VII.2015; 6 우, same locality, pitfall traps, 513.VII.2015; mountain tundra, goltsy: 6 o , sifting, 5-9.VII.2015; same locality, $1 \sigma^{\top}, 4$ 우 , pitfall traps, 6-15.VII.2015.

Azon. Tyylaakh, steep slopes of Lena River bank: $1 \sigma^{7}$, in soil, under stones, 11.VII.2015.

REMARKS. This species was hitherto known from Ketalakh Lake (Map, No. 6) [Kulczyński, 1908], from Tiksi (Map, No. 2) [Eskov, 1987], as well as from Bykovsky Peninsula (Map, No. 3) [Marusik et al., 1993].

RANGE. Siberian arcto-boreo-montane.

\section{Hilaira herniosa (Thorell, 1875)}

REMARKS. Known from the Lena River Valley: TitAry \& Tas-Ary islands, Bulun, Kyusyur \& Beris River (Map, No. 8, 10 \& 11) [Marusik et al., 1993].

RANGE. Holarctic boreal.

Hilaira hyperborea Kulczyński, 1908, comb.revalid.

MATERIAL. Intra. Tiksi, moss-lichen communities in rocky debris: 1 + sifting, 20.VII.2015; rocky outcrops in tundra: $1 \sigma^{7}$ among stones, 18.VII.2015; Polyarka, rocky outcrops in tundra: 1 + , among stones, 21.VII.2015.

Mountain. Tyylaakh, mountain tundra, goltsy: 1 , sifting, 9.VII.2015.

REMARKS. The species was originally described based on a female from Bykovsky Peninsula as Hilaira hyperborea (Map, No. 3) [Kulczyński, 1908]. Later, Eskov transferred this species into the genus Sciastes Bishop et Crosby, 1938 , with many subsequent citations within the latter genus [see Eskov, 1994]. According to the conformation of the genitalia of both sexes, as well as the chaetotaxy, the species undoubtedly belongs to the genus Hilaira Simon, 1884, and seems to be most similar to the East Siberian - Far Eastern H. devitata Eskov, 1987. Besides Bykovsky Peninsula, $H$. hyperborea is also known from Tiksi (Map, No. 2) [Marusik et al., 1993, as S. hyperboreus].

RANGE. Middle Siberian arcto-alpine.

Hilaira incondita (L. Koch, 1879)

MATERIAL. Intra. Tyylaakh, dwarf willow communities: 1 $\circ$, sifting, 12.VII.2015; $41 \sigma^{7} \sigma^{7}$, same locality, 10-13.VII.2015; 14 ऽ ऽ, same locality, 13-16.VII.2015.

Azon. Tyylaakh, bank of Lena River: 2 $\circ$, under stones, 7.VII.2015; $10 \sigma^{7} \sigma^{7}$, same locality, pitfall traps, 10-16.VII.2015 pebble bank of Tylakh-Yuryage River: $1 \sigma^{7}$, under stones, 7.VII.2015.

Antro. Tyylaakh, grass associations: $7 \sigma^{7} \sigma^{7}$, pitfall traps, 14 16.VII.2015. Tiksi, under stones and rubbish: 14 우, 17.VII.2015.

REMARKS. This species was hitherto known from Tiksi (Map, No. 2) [Eskov, 1987].

RANGE. Siberian-Nearctic arcto-boreal.

Hilaira nivalis Holm, 1937

MATERIAL. Zonal. Tyylaakh, hummocky tundra: $2+9$, sifting, 7.VII.2015; env. of Tylakh-Kyuel Lake, undershrub-lichenmoss tundra: 1 , sifting, 10.VII.2015.

Intra. Tyylaakh, dwarf willow communities: $1+$, pitfall traps, 7-10.VII.2015; 3 O $^{7}$, same locality, 13-16.VII.2015; moss-sedge swamp: 5 우, sifting moss and litter, 14.VII.2015; swamped bank of lake: 1 , in Sphagnum, sifting, 8.VII.2015; Astragalus-mossDryas communities: 2 우 , sifting, 10.VII.2015. Tiksi, moss-sedge swamp: 1 ऊ , 2 우, in Sphagnum, sifting, 20.VII.2015; moss-sedge swamp: 1 ऽ', 7 우, sifting moss and litter, 20.VII.2015.
Azon. Tyylaakh, steep slopes of Lena River bank: $2 \sigma^{7}$, in soil, under stones, 11.VII.2015. Bank of Lena River: 1 , under drift woods, 11.VII.2015.

Antro. Tyylaakh, grass associations: $1 \sigma^{7}$, pitfall traps, 1416.VII.2015. Tiksi, under stones and rubbish: 2 +9, 17.VII.2015.

REMARKS. This species was hitherto known from Ketalakh Lake (Map, No. 6) [Kulczyński, 1908, as H. glacial$i s$, and from Tiksi (Map, No. 2) [Eskov, 1987].

RANGE. West Siberian arctic.

\section{Hilaira proletaria (L. Koch, 1879)}

MATERIAL. Intra. Tyylaakh, moss-sedge swamp: 17 † , sifting moss and litter, 12-15.VII.2015; swamped bank of lake: 19 , in Sphagnum, sifting, 8.VII.2015. Tiksi, moss-sedge swamp: 1 , in Sphagnum, sifting, 20.VII.2015; moss-sedge swamp: 3 o $\sigma^{7}, 3$ 우, sifting moss and litter, 20.VII.2015.

REMARKS. This species was hitherto known from Tiksi (Map, No. 2) [Marusik et al., 1993].

RANGE. Siberian-West Nearctic arcto-boreal.

\section{Holminaria pallida Eskov, 1991}

MATERIAL. Intra. Tiksi, rocky outcrops in tundra: $1 \sigma^{7}$, under stones, 18.VII.2015.

RANGE. Siberian boreal.

\section{Hybauchenidium aquilonare (L. Koch, 1879)}

MATERIAL. Zonal. Tyylaakh, hummocky tundra: 4 우, pitfall traps, 7-16.VII.2015. Tiksi, lichen-moss-dwarf birch tundra: 2 O' $\sigma^{\top}, 3$ 우, sifting, 20.VII.2015.

Intra. Tyylaakh, dwarf willow communities: $1 \sigma^{\text {r, }}$, sifting, 7.VII.2015; $1 \sigma^{7}$, same locality, pitfall traps, 10-13.VII.2015; Astragalus-moss-dwarf willow communities: $3 \sigma^{7} \sigma^{7}$, sifting, 15.VII.2015; Astragalus-moss-Dryas communities: $1 \bigcirc^{7}, 5$ 우, sifting, 15.VII.2015; 2 우, same locality, pitfall traps, 12-15.VII.2015; moss-sedge swamp: 1 , sifting moss and litter, 15.VII.2015. Tiksi, moss-sedge swamp: $1 \sigma^{\top}, 2$ 우, in Sphagnum, sifting, 20.VII.2015.

REMARKS. This species was hitherto known from the Antyngilkan River (Map, No. 7) [Kulczyński, 1908, as Gongylidium septentrionale], Tiksi \& Bykovsky Peninsula (Map, No. 2 \& 3), as well as from the Lena River Valley: Tit-Ary \& Tas-Ary islands (Map, No. 8) [Marusik et al., 1993].

RANGE. Siberian-West Nearctic arcto-boreal.

Hypomma bituberculatum (Wider, 1834)

REMARKS. Known from Bulun, Kyusyur \& Beris River (Map, No. 10 \& 11) [Marusik et al., 1993].

RANGE. Palaearctic polyzonal.

Hypselistes jacksoni (O. Pickard-Cambridge, 1902)

MATERIAL. Zonal. Tyylaakh, hummocky tundra: 3 우, pitfall traps, 7-16.VII.2015. Tiksi, lichen-moss-dwarf birch tundra: 1 o', 3 우, sifting, 20.VII.2015.

Intra. Tyylaakh, dwarf willow communities: $1+$, pitfall traps, 13-16.VII.2015; Astragalus-Dryas communities: 3 우, sifting, 15.VII.2015. Tiksi, moss-sedge swamp: $2 \sigma^{7} \sigma^{7}$, sifting moss and litter, 20.VII.2015.

REMARKS. This species was hitherto known from Tiksi (Map, No. 2) as Hypselistes semiflavus [Marusik et al., 1993].

RANGE. Palaearctic-West Nearctic polyzonal.

Incestophantes incestus (L. Koch, 1879)

REMARKS. Known from the Lena River Valley: Bulun, Kyusyur \& Beris River (Map, No. 10 \& 11) [Marusik et al., 1993].

RANGE. Siberian boreal. 

1987)

Incestophantes laricetorum (Tanasevitch et Eskov,

REMARKS. Known from the Lena River Valley: Bulun, Kyusyur \& Beris River (Map, No. 10 \& 11) [Marusik et al., 1993].

RANGE. Siberian boreal.

\section{Islandiana cristata Eskov, 1987}

REMARKS. Known from the Lena River Valley: Bulun, Kyusyur \& Beris River (Map, No. 10 \& 11) [Marusik et al., 1993].

RANGE. Siberian-West Nearctic arcto-alpine.

Lepthyphantes nenilini Tanasevitch, 1987

REMARKS. Known from the Lena River Valley: Bulun, Kyusyur \& Beris River (Map, No. 10 \& 11) [Marusik et al., 1993].

RANGE. Siberian arcto-alpine.

\section{Masikia indistincta (Kulczyński, 1908)}

MATERIAL. Zonal. Tyylaakh, hummocky tundra: 4 우, sifting, 7.VII.2015; $3 \bigcirc^{7} \sigma^{7}$, same locality, pitfall traps, 7-16.VII.2015 undershrub-lichen-moss tundra: $1 \mathrm{O}^{7}$, pitfall traps, 5-15.VII.2015.

Intra. Tyylaakh, dwarf willow communities: 2 우, sifting, 7.VII.2015; $20^{7} \sigma^{7}, 4$ 오, same locality, pitfall traps, 10-16.VII.2015; moss-sedge swamp: $6 O^{7} \sigma^{7}, 25 \circ+$, sifting moss and litter 15.VII.2015; swamped bank of lake: 10 우, in Sphagnum, sifting, 8.VII.2015.

Azon. Tyylaakh, bank of Lena River: $1 \sigma^{7}$, pitfall traps, $7-$ 16.VII.2015.

Antro. Tyylaakh, grass associations: $1 \mathrm{O}^{7}$, pitfall traps, 14 16.VII.2015.

REMARKS. This species was hitherto known from seashore near the Kharaulakh River (Map, No. 5) [Kulczyński, 1908, as Macrargus (?) indistinctus].

RANGE. Siberian-Nearctic arctic.

\section{Mecynargus monticola (Holm, 1943)}

REMARKS. Known from the Lena River Valley: Bulun, Kyusyur \& Beris River (Map, No. 10 \& 11) [Marusik et al., 1993].

RANGE. Fennoscandian-Siberian-West Nearctic boreal.

Mecynargus paetulus (O. Pickard-Cambridge, 1875)

REMARKS. Known from the Lena River Valley: Bulun, Kyusyur \& Beris River (Map, No. 10 \& 11) [Marusik et al., 1993].

RANGE. Holarctic boreo-nemoral.

\section{Mecynargus tundricola Eskov, 1988}

MATERIAL. Zonal. Tyylaakh, hummocky tundra: $3 \sigma^{7} \sigma^{7}, 7$ 우, sifting, 7.VII.2015; 1 0े, 9 우, same locality, pitfall traps, 716.VII.2015; undershrub-lichen-moss tundra: $1 \sigma^{7}, 3$ 웅, sifting, 5.VII.2015; 2 우, same locality, pitfall traps, 6-16.VII.2015; env.

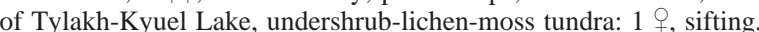
10.VII.2015. Tiksi, hummocky tundra: 3 우, sifting, 20.VII.2015; lichen-moss-dwarf birch tundra: $1 \sigma^{7}$, sifting, 20.VII.2015.

Intra. Tyylaakh, dwarf willow communities: $10^{7}$, pitfall traps, 7-10.VII.2015; Astragalus-moss-dwarf willow communities: 8 우, sifting, 15.VII.2015; Astragalus-moss-Dryas communities: 2 우, sifting, 10-12.VII.2015.

Mountain. Tyylaakh, mountain tundra on rocky terrace: 8 우, sifting, 5-9.VII.2015; $2 \mathrm{O}^{7} \mathrm{O}^{7}, 8$ 우, same locality, pitfall traps, 59.VII.2015, mountain tundra, goltsy: $3 \sigma^{7} \sigma^{7}, 15$ 웅, sifting, 513.VII.2015; $1 \mathrm{O}^{\top}$, same locality, pitfall traps, 6-15.VII.2015.

Azon. Tyylaakh, bank of Lena River: $5 O^{7} O^{7}, 7$ 우, under drift woods, 10-12.VII.2015.

RANGE. Siberian arcto-boreal.

Mughiphantes sobrius (Thorell, 1872)

MATERIAL. Zonal. Tyylaakh, hummocky tundra: 3 우, sifting, 7.VII.2015; 5 O $^{7}$, 9 के, same locality, pitfall traps, 716.VII.2015; undershrub-lichen-moss tundra: 1 + , pitfall traps, 515.VII.2015; dwarf willow communities: 19 , sedges, sweeping, 12.VII.2015; moss-sedge swamp: 1 \% , sifting moss and litter, 15.VII.2015; Astragalus-moss-Dryas communities: 1 , sifting, 10-15.VII.2015. Tiksi, rocky outcrops in tundra: $1+$, under stones, 18.VII.2015; lichen-moss-dwarf birch tundra: 1 , sifting, 20.VII.2015; lichen-moss-dwarf willow tundra: 1 , sifting, 20.VII.2015; moss-sedge swamp: $1+$, sifting moss and litter, 20.VII.2015.

Mountain. Tyylaakh, mountain tundra on rocky terrace: $10^{7}$, 1 오 , sifting, 9.VII.2015; 3 우오 same locality, pitfall traps, 59.VII.2015; mountain tundra, goltsy: 1 + , sifting, 9.VII.2015; 6 $O^{\top} O^{\top}, 6$ 우, same locality, pitfall traps, 5-15.VII.2015.

Azon. Tyylaakh, steep slopes of Lena River bank: $30^{7} \mathrm{O}^{7}$, in soil, under stones, 11.VII.2015; bank of Lena River: 8 우, under drift woods, 11.VII.2015; $1 \mathrm{O}^{7}$, same locality, pitfall traps, 14-16.VII.2015.

Antro. Tiksi, under stones and rubbish: 3 o+ , 17.VII.2015.

REMARKS. This species was hitherto known from Tiksi (Map, No. 2) and from the Lena River Valley: Tit-Ary \& Tas-Ary islands (Map, No. 8) [Marusik et al., 1993, as Lepthyphantes s.].

RANGE. Palaearctic arctic.

Oreoneta alpina (Eskov, 1987)

REMARKS. Known from Tiksi (Map, No. 2) [Marusik et al., 1993, as Hilaira a.].

RANGE. Middle \& East Siberian arcto-alpine.

Oreoneta leviceps (L. Koch, 1879)

REMARKS. Known from the Lena River Valley: TitAry \& Tas-Ary islands (Map, No. 8) [Marusik et al., 1993, as Hilaira l.].

RANGE. Siberian-Nearctic arcto-boreal.

\section{Paraglyphesis polaris Eskov, 1991}

MATERIAL. Zonal. Tyylaakh, hummocky tundra: $3 \sigma^{7} \sigma^{7}, 8$ 우, sifting, 7.VII.2015; undershrub-lichen-moss tundra: $10^{7}, 3$ 우, sifting, 5.VII.2015; env. of Tylakh-Kyuel Lake, undershrub-lichenmoss tundra: $1 \sigma^{7}, 1$ o, sifting, 10.VII.2015. Tiksi, hummocky tundra: $1 \sigma^{\top}, 2$ 우, sifting, 20.VII.2015; lichen-moss-dwarf birch tundra: $3 \sigma^{7} \sigma^{7}, 3$ o 9 , sifting, 20.VII.2015; $1 \sigma^{7}$, same locality, pitfall traps, 18-20.VII.2015; lichen-moss-dwarf willow tundra: 3 $\mathrm{O}^{\top} \mathrm{O}^{\top}, 3$ 우, sifting, 20.VII.2015.

Intra. Tyylaakh, swamped bank of lake: $1 \%$, in Sphagnum, sifting, 8.VII.2015; Astragalus-moss-Dryas communities: 2 우, sifting, 10-15.VII.2015. Tiksi, moss-sedge swamp: 1 \% , sifting moss and litter, 20.VII.2015.

Mountain. Tyylaakh, mountain tundra, goltsy: $1 \sigma^{7}$, sifting, 13.VII.2015.

RANGE. Siberian arcto-boreal.

Pelecopsis mengei (Simon, 1884)

REMARKS. Known from the Lena River Valley: Bulun, Kyusyur \& Beris River (Map, No. 10 \& 11) [Marusik et al., 1993].

RANGE. Holarctic polyzonal.

Pelecopsis parallela (Wider, 1834)

MATERIAL. Zonal. Tyylaakh, hummocky tundra: $1+$, pitfall traps, 7-16.VII.2015. 
Intra. Tyylaakh, moss-sedge swamp: $1 \sigma^{7}, 1$, sifting moss and litter, 12.VII.2015.

Mountain. Tyylaakh, mountain tundra on rocky terrace: $1 \sigma^{7}$, sifting, 5.VII.2015; 1 +, same locality, pitfall traps, 5-9.VII.2015.

Antro. Tyylaakh, grass associations: $2 \sigma^{7} \sigma^{7}$, pitfall traps, 14 16. VII.2015.

RANGE. Palaearctic polyzonal.

Perro polaris (Eskov, 1986)

REMARKS. Known from Tiksi (Map, No. 2) [Eskov, 1986].

RANGE. Siberian-West Nearctic arcto-boreal.

Poeciloneta pallida Kulczyński, 1908

MATERIAL. Intra. Tiksi, moss-lichen communities in rocky debris: $1 \sigma^{7}$, under stones, 18-20.VII.2015; rocky outcrops in tundra: $6 \bigcirc^{7} \sigma^{7}, 18$ 우, among stones, 18.VII.2015; Polyarka, rocky outcrops in tundra: $1+$, among stones, 21.VII.2015.

Mountain. Tyylaakh, mountain tundra, goltsy: 3 of, sifting, 9.VII.2015.

REMARKS. The species was originally described from the Elidep River mouth (Map, No. 6) [Kulczyński, 1908], and later was recorded from Tiksi, as well as from the Lena River Valley: Tit-Ary and Tas-Ary islands, Bulun, Kyusyur and Beris River (Map, No. 8, 10 \& 11) [Marusik et al., 1993].

RANGE. Siberian arcto-alpine.

Praestigia groenlandica Holm, 1967

MATERIAL. Zonal. Tyylaakh, hummocky tundra: $1 \sigma^{7}$, pitfall traps, 7-16.VII.2015.

Intra. Tyylaakh, dwarf willow communities: $1+$, pitfall traps, 10-13.VII.2015. Tiksi, moss-sedge swamp: $1 \overbrace{}^{7}$, pitfall traps, 18 20.VII.2015; moss-sedge swamp: 4 우, sifting moss and litter, 20.VII.2015.

REMARKS. This species was hitherto known from the Lena River Valley: Bulun, Kyusyur and Beris River (Map, Nos. 10, 11) [Marusik et al., 1993]. We consider P. groenlandica as a circumpolar species, unlike Marusik et al. [2008] who separated several species on its basis, which are associated with different sectors of the Arctic.

RANGE. Siberian-Nearctic arctic.

Proislandiana pallida (Kulczyński, 1908)

MATERIAL. Intra. Tiksi, Polyarka, rocky outcrops in tundra: 2 우, among stones, 21.VII.2015.

REMARKS. The species was originally described from the Ketalakh Lake (Map, No. 6) [Kulczyński, 1908, as Microneta (?) p.].

RANGE. Siberian arcto-alpine.

Scotinotylus alpigena (L. Koch, 1869)

REMARKS. Known from the Lena River Valley: Bulun, Kyusyur \& Beris River (Map, No. 10 \& 11) [Marusik et al. 1993, as S. alpigenus].

RANGE. Palaearctic boreo-nemoral.

Scotinotylus alpinus (Banks, 1896)

REMARKS. Known from the Lena River Valley: Bulun, Kyusyur \& Beris River (Map, No. 10 \& 11) [Marusik et al., 1993].

RANGE. Siberian-West Nearctic boreo-montane.

Semljicola angulatus (Holm, 1963)

REMARKS. Known from the Lena River Valley: Bulun, Kyusyur \& Beris River (Map, No. 10 \& 11) [Marusik et al., 1993].
RANGE. Fennoscandian-Siberian boreal.

\section{Semljicola arcticus (Eskov, 1989)}

MATERIAL. Zonal. Tyylaakh, hummocky tundra: 19 , pitfall traps, 7-16.VII.2015; undershrub-lichen-moss tundra: 1 q, sifting, 5.VII.2015.

Mountain. Tyylaakh, mountain tundra on rocky terrace: 290 , pitfall traps, 6-13.VII.2015; 1 \% , sifting, 5.VII.2015; mountain tundra, goltsy: 1 , pitfall traps, 5-9.VII.2015.

RANGE. Siberian arctic.

Semljicola barbiger (L. Koch, 1879)

MATERIAL. Intra. Tiksi, moss-sedge swamp: 4 우, sifting moss and litter, 20.VII.2015.

RANGE. Fennoscandian-Siberian arcto-boreo-montane.

Semljicola simplex (Kulczyński, 1908)

MATERIAL. Zonal. Tiksi, lichen-moss-dwarf birch tundra: 1 $0^{7}, 3$ o, , sifting, 20.VII.2015.

Intra. Tyylaakh, dwarf willow communities: $1 \sigma^{7}, 3+0$, pitfall traps, 10-13.VII.2015; Astragalus-moss-dwarf willow communities: 1 , sifting, 15.VII.2015; moss-sedge swamp: $1 \sigma^{7}, 3$ 우, sifting moss and litter, 15.VII.2015; swamped bank of lake: $1 \sigma^{\top}, 1$ +, in Sphagnum, sifting, 8.VII.2015.

Mountain. Tyylaakh, mountain tundra on rocky terrace: 1 오 pitfall traps, 9-13.VII.2015; mountain tundra, goltsy: $2 \sigma^{\top} \sigma^{\top}, 8$ 우, pitfall traps, 6-15.VII.2015.

Azon. Tyylaakh, bank of Lena River: 12 우, pitfall traps, 1014.VII.2015.

Antro. Tyylaakh, grass associations: $1 \overbrace{}^{7}, 2$ 우, pitfall traps, 14-16.VII.2015. Tiksi, under stones and rubbish: 2 오, 17.VII.2015.

REMARKS. The species was originally described from the "Kharaulakh mountains" without locality [Kulczyński, 1908, as Styloctetor (?) s.], but based on the date of collection it is not so far from the Elidep River mouth..

RANGE. Siberian arcto-alpine.

Sibirocyba incerta (Kulczyński, 1916)

MATERIAL. Azon. Tyylaakh, pebble bank of Tylakh-Yuryage River: $15 \sigma^{7} \sigma^{7}, 38$ 우, under stones, 7.VII.2015; 5 우, same locality, 10.VII.2015.

RANGE. Siberian arcto-boreo-montane.

Silometopoides pampia (Chamberlin, 1948)

MATERIAL. Zonal. Tyylaakh, undershrub-lichen-moss tundra: $1 \sigma^{T}$, sifting, 5.VII.2015. Mountain. Tyylaakh, mountain tundra, goltsy: 1 \% , sifting, pitfall traps, 6-15.VII.2015.

RANGE. Siberian-West Nearctic arctic.

Silometopoides sphagnicola Eskov et Marusik, 1992

MATERIAL. Zonal. Tyylaakh, hummocky tundra: 2 우, pitfall traps, 7-16.VII.2015.

Intra. Tyylaakh, dwarf willow communities: $1 q$, pitfall traps, 13-16.VII.2015; moss-sedge swamp: 1 을 sifting moss and litter, 15.VII.2015. Tiksi, moss-sedge swamp: 2 क्+, sifting moss and litter, 20.VII.2015.

REMARKS. This species was hitherto known from the Lena River Valley: Tit-Ary \& Tas-Ary islands (Map, No. 8) [Marusik et al., 1993].

RANGE. Siberian arcto-boreal.

Tubercithorax subarcticus (Tanasevitch, 1984)

MATERIAL. Zonal. Tiksi, hummocky tundra: 1 , sifting, 20.VII.2015. 
Intra. Tiksi, moss-sedge swamp: $1+$, sifting moss and litter, 20.VII.2015; lichen-moss-dwarf birch tundra: $1 \sigma^{\top}, 7$ \% , sifting, 20.VII.2015; $6 \bigcirc^{7} \circlearrowleft^{7}$, same locality, pitfall traps, 18-20.VII.2015; lichen-moss-dwarf willow tundra: $1 \sigma^{7}, 7$ 우, sifting, 20.VII.2015.

REMARKS. This species was hitherto known from the Lena River Valley: Tit-Ary \& Tas-Ary islands (Map, No. 8) [Marusik et al., 1993].

RANGE. Siberian arcto-boreal.

\section{Wabasso millidgei Eskov, 1988}

MATERIAL. Azon. Tyylaakh, bank of Lena River: $1 \sigma^{\gamma}$, pitfall traps, 7-16.VII.2015.

RANGE. Siberian arcto-boreal. 1873)

Walckenaeria karpinskii (O. Pickard-Cambridge,

REMARKS. Known from the Lena River Valley: Bulun, Kyusyur \& Beris River (Map, No. 10 \& 11) [Marusik et al., 1993].

RANGE. Fennoscandian-Siberian-Nearctic boreal.

Walckenaeria korobeinikovi Esyunin et Efimik, 1996 Figs 21-22.

MATERIAL. Zonal. Tyylaakh, hummocky tundra: 5 우, sifting, 7.VII.2015; $3 \sigma^{7} \sigma^{7}$, same locality, pitfall traps, 7-16.VII.2015. Tiksi, hummocky tundra: 1 q, sifting, 20.VII.2015; moss-sedge swamp: 1 , in Sphagnum, sifting, 20.VII.2015; lichen-moss-dwarf willow tundra: 19 , sifting, 20.VII.2015.

Intra. Tyylaakh, dwarf willow communities: 4 + , pitfall traps, 7-16.VII.2015. Tiksi, Polyarka, rocky outcrops in tundra: $1+$ among stones, 21.VII.2015.

Mountain. Tyylaakh, mountain tundra on rocky terrace: 1 , sifting, 9.VII.2015; 1 ऽ, 4 $\circ$, same locality, pitfall traps, 5 9.VII.2015; mountain tundra, goltsy: 1 \% , sifting, 5-9.VII.2015.

Azon. Tyylaakh, bank of Lena River: 1 + , under stones, 11.VII.2015

Antro. Tiksi, under stones and rubbish: 1 \% , 17.VII.2015.

REMARKS. This species was hitherto known as Walckenaeria clavicornis (Emerton, 1882) from Tiksi (Map, No. 2), as well as from the Lena River Valley: Bulun, Kyusyur and Beris River (Map, Nos. 10, 11) [Marusik et al., 1993]. According to the male cymbium conformation, all specimens collected in the region belong to $W$. korobeinikovi (see Figs 21-22).

RANGE. Palaearctic arcto-boreal.

Fam. Lycosidae (11)

Alopecosa hirtipes (Kulczyński, 1908)

MATERIAL. Intra. Tiksi, Polyarka, rocky outcrops in tundra: 1 , 1 ○ subad., 1 juv., among stones, 21.VII.2015.

REMARKS. The species was hitherto known from Khoptolokh River (Map, No. 4), the "Kharaulakh Mountains" (without locality) [Kulczyński, 1908, as Tarentula h.], as well as from the Lena River Valley: Tit-Ary \& Tas-Ary islands (Map, No. 8) [Marusik et al., 1993].

RANGE. Siberian-Nearctic arctic [Dondale, Redner, 1990; Tanasevitch, Koponen, 2007; Paquin et al., 2010].

\section{Alopecosa mutabilis (Kulczyński, 1908)}

MATERIAL. Zonal. Tyylaakh, hummocky tundra: 2 90 , pitfall traps, 7-16.VII.2015; undershrub-lichen-moss tundra: $31 \bigcirc^{7} \sigma^{7}$ 10 우, pitfall traps, 5-15.VII.2015. Tiksi, hummocky tundra: 1 juv., pitfall traps, 18-20.VII.2015; lichen-moss-dwarf birch tun- dra: 2 oㅇ, sifting, 20.VII.2015; 3 juv., same locality, pitfall traps, 18-20.VII.2015; moss-lichen communities in rocky debris: $1 \sigma^{7}$, pitfall traps, 18-20.VII.2015.

Intra. Tyylaakh, Astragalus-moss-Dryas communities: 1 , pitfall traps, 12-15.VII.2015. Tiksi, moss-lichen communities in rocky debris: 5 juv., sifting, 20.VII.2015; Polyarka, rocky outcrops in tundra: 10 juv., among stones, 21.VII.2015.

Mountain. Tyylaakh, mountain tundra on rocky terrace: $1 \sigma^{7}$, pitfall traps, 5-9.VII.2015.

REMARKS. This species was hitherto known from the "Kharaulakh Mountains" (without locality) \& the Kharaulakh River Valley (Map, No. 7) [Kulczyński, 1908, as Tarentula m.], from Tiksi (Map, No. 2), from the Lena River Valley: Tit-Ary \& Tas-Ary islands (Map, No. 8), as well as wrongly as $A$. albostriata from Bulun, Kyusyur \& Beris River (Map, No. 10 \& 11) [Marusik et al., 1993] (Marusik, pers. com., 2016).

RANGE. Siberian-West Nearctic arctic. [Esyunin, Efimik, 1996; Dondale et al., 1997; Paquin et al., 2010; Mikhailov, 2013].

\section{Alopecosa sibirica (Kulczyński, 1908)}

REMARKS. This species was hitherto known from the Elidep River mouth and Ketalakh Köle (Map, No. 6) [Kulczyński, 1908, as Lycosa s.].

RANGE. East Palaearctic arcto-boreal [Marusik et al., 2000].

Alopecosa solivaga borea (Kulczyński, 1908)

REMARKS. Known from the Lena River Valley: TitAry \& Tas-Ary islands (Map, No. 8), as well as from Bulun, Kyusyur \& Beris River (Map, No. 10 \& 11) as Alopecosa borea [Marusik et al., 1993].

RANGE. Arcto-boreal [Esyunin, Efimik, 1996; Marusik et al., 2000].

\section{Arctosa alpigena (Doleschall, 1852)}

REMARKS. Known from the Lena River Valley: TitAry \& Tas-Ary islands (Map, No. 8), as well as from Bulun, Kyusyur \& Beris River (Map, No. 10 \& 11) [Marusik et al., 1993, as Tricca a.].

RANGE. Holarctic arcto-boreal [Nekhaeva, 2015].

Pardosa algens (Kulczyński, 1908)

REMARKS. Known from the "Kharaulakh Mountains" (without locality) [Kulczyński, 1908, as Lycosa a.].

RANGE. Siberian-Nearctic arcto-boreal [Kronestedt, 1986; Dondale, Redner, 1990; Paquin et al., 2010; Mikhailov, 2013].

Pardosa eiseni (Thorell, 1885)

REMARKS. Known from Tiksi (Map, No. 2) [Marusik et al., 1993].

RANGE. Palaearctic arcto-boreal [Nekhaeva, 2015].

Pardosa groenlandica (Thorell, 1872)

REMARKS. Known from the Lena River Valley, Chekurovka (Map, No. 9) [Marusik et al., 1993]. 


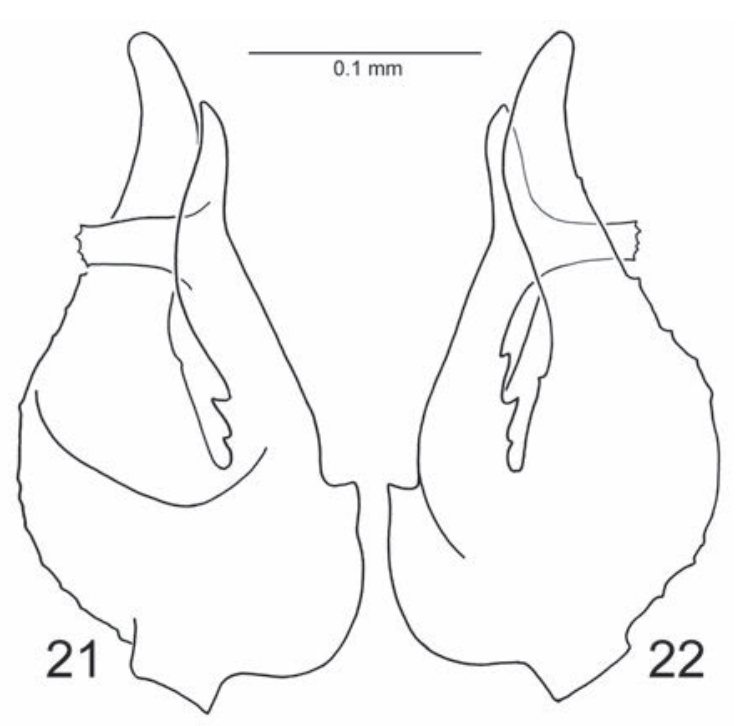

Figs. 21-22. Left palpal tibia of Walckenaeria korobeinikovi Esyunin et Efimik, 1996, different aspects, specimen from Tyylaakh, mountain tundra.

Рис. 21-22. Голень левой пальпы Walckenaeria korobeinikovi Esyunin et Efimik, 1996, различные аспекты, экземпляр из Тыылаах, горная тундра.

RANGE. East Siberian-Nearctic arcto-boreal [Dondale et al., 1997; Paquin et al., 2010; Mikhailov, 2013; Slowik, Sikes, 2013].

\section{Pardosa septentrionalis (Westring, 1861)}

MATERIAL. Zonal. Tyylaakh, hummocky tundra: $155 \sigma^{7} \sigma^{7}, 26$ $\bigcirc$, pitfall traps, 7-16.VII.2015; undershrub-lichen-moss tundra: 27 $\mathrm{O}^{7} \mathrm{O}^{7}$, pitfall traps, 5-15.VII.2015; env. of Tylakh-Kyuel Lake, undershrub-lichen-moss tundra: $2 \sigma^{7} \sigma^{7}$, by hands, 10.VII.2015. Tiksi, hummocky tundra: $3 \sigma^{7} \sigma^{7}$, pitfall traps, 18-20.VII.2015; lichenmoss-dwarf birch tundra: $8 \mathrm{O}^{7} \sigma^{7}$, pitfall traps, 18-20.VII.2015.

Intra. Tyylaakh, dwarf willow communities: $142{\sigma^{7}}^{7}, 3$ 우, pitfall traps, 7-16.VII.2015; Astragalus-moss-dwarf willow communities: $3 \sigma^{7} \sigma^{7}$, pitfall traps, 12-15.VII.2015; Astragalus-mossDryas communities: $20 \sigma^{7} \sigma^{7}, 1$, pitfall traps, 12-15.VII.2015. Tiksi, moss-sedge swamp: $10^{7}, 2$ 우, pitfall traps, 20-22.VII.2015; moss-sedge swamp: $2 \circlearrowleft^{7} \sigma^{7}$, pitfall traps, 18-20.VII.2015; rocky outcrops in tundra: 1 , , under stones, 18.VII.2015.

Mountain. Tyylaakh, mountain tundra on rocky terrace: $2+q$, sifting, 9.VII.2015; $10 \overbrace{}^{7} \bigcirc^{7}, 28$ 우, same locality, pitfall traps, 513.VII.2015; mountain tundra, goltsy: $43 \bigcirc^{7} \sigma^{7}, 7$ 9 , pitfall traps, 6-15.VII.2015.

Azon. Tyylaakh, bank of Lena River: $10^{7}$, pitfall traps, 10 14.VII.2015.

Antro. Tyylaakh, grass associations: $8 \odot^{7} \sigma^{7}$, pitfall traps, 14 16.VII.2015.

REMARKS. This species was hitherto known from Tiksi (Map, No. 2), as well as from the Lena River Valley: TitAry \& Tas-Ary islands (Map, No. 8) [Marusik et al., 1993].

RANGE. Fennoscandian-Siberian arcto-boreal [Tanasevitch, Koponen, 2007].

\section{Pardosa tesquorum (Odenvall, 1901)}

REMARKS. Known from the Lena River Valley: Bulun, Kyusyur \& Beris River (Map, No. 10 \& 11) [Marusik et al., 1993].
RANGE. Siberian-Nearctic arcto-boreal [Dondale, Redner, 1990; Marusik et al., 2000; Paquin et al., 2010; Kronestedt, Marusik, 2011].

\section{Sibirocosa sibirica (Kulczyński, 1908)}

REMARKS. The species was originally described from the Elidep River mouth as Lycosa s. (Map, No. 6) [Kulczyński, 1908, as Lycosa s.], and latter it was registered from the Lena River Valley: Tit-Ary \& Tas-Ary islands (Map, No. 8) [Marusik et al., 1993, as Acantholycosa s.].

RANGE. East Siberian arcto-boreal [Marusik et al., 2003; Omelko, Marusik, 2013].

Fam. Philodromidae (2)

Philodromus alascensis Keyserling, 1884

REMARKS. Known from Tiksi (Map, No. 2), as well as from the Lena River Valley: Bulun, Kyusyur \& Beris River (Map, No. 10 \& 11) [Marusik et al., 1993].

RANGE. Siberian-Nearctic polyzonal [Dondale, Redner, 1978; Marusik et al., 2000; Szita, Logunov, 2008; Paquin et al., 2010].

Thanatus arcticus Thorell, 1872

REMARKS. Known from Tiksi (Map, No. 2), as well as from Chekurovka, Lena River Valley (Map, No. 9) [Marusik et al., 1993].

RANGE. Holarctic arcto-boreal [Dondale, Redner, 1978; Marusik et al., 2006; Paquin et al., 2010].

Fam. Tetragnathidae (2)

Pachygnatha clercki Sundevall, 1823

MATERIAL. Zonal. Tyylaakh, hummocky tundra: $1 \sigma^{7}, 1$ juv., pitfall traps, 7-10.VII.2015.

Intra. Tyylaakh, dwarf willow communities: $1+$, pitfall traps, 10-13.VII.2015; moss-sedge swamp: 1 \%, 3 juv., sifting moss and litter, 15.VII.2015. Tiksi, moss-sedge swamp: $4 \sigma^{7} \sigma^{7}, 4$, 9 , sifting moss and litter \& pitfall traps, 18-20.VII.2015.

Azon. Tiksi, pebble bank of Laptev Sea: 1 \% , among stones, 18.VII.2015.

Antro. Tyylaakh, grass associations: 3 우, pitfall traps, 1116.VII.2015.

RANGE. Holarctic polyzonal [Marusik et al., 2000; Tanasevitch, Koponen, 2007; Paquin et al., 2010].

\section{Tetragnatha extensa (Linnaeus, 1758)}

REMARKS. Known from the Lena River Valley: TitAry \& Tas-Ary islands (Map, No. 8) [Marusik et al., 1993], and from Bulun (Map, No. 10) as T. pinicola (L. Koch, 1870) [Marusik et al., 1993].

RANGE. Holarctic polyzonal [Nekhaeva, 2015].

Fam. Theridiidae (4)

Robertus lyrifer Holm, 1939

REMARKS. Known from the Lena River Valley: Bulun, Kyusyur \& Beris River (Map, No. 10 \& 11) [Marusik et al., 1993].

RANGE. Holarctic boreal [Nekhaeva, 2015].

Steatoda castanea (Clerck, 1758)

MATERIAL. Antro. Tiksi: $1+$, in flat, 18.VII.2015. 
RANGE. Holarctic polyzonal [Esyunin, Efimik, 1996; Paquin et al., 2010; Mikhailov, 2013].

\section{Thymoites bellissimus (L. Koch, 1879)}

MATERIAL. Mountain. Tyylaakh, mountain tundra on rocky terrace: $1 O^{7}$, sifting, 9.VII.2015; mountain tundra, goltsy: $5 \sigma^{7} \sigma^{7}$, 12 ㅇ, 20 juv., sifting, 9.VII.2015; $1 \mathrm{O}^{7}$, same locality, pitfall traps, 6-15.VII.2015.

Antro. Tiksi, under stones and rubbish: 1 , 17.VII.2015.

RANGE. Palaearctic boreal [Esyunin, Efimik, 1996; Marusik et al., 2000; Mikhailov, 2013].

Thymoites oleatus (L. Koch, 1879)

REMARKS. Known from Tiksi (Map, No. 2), and from the Lena River Valley: Tit-Ary \& Tas-Ary islands (Map, No. 8) [Marusik et al., 1993].

RANGE. Siberian-Nearctic arcto-boreal [Dondale et al., 1997; Marusik et al., 2006; Tanasevitch, Koponen, 2007; Paquin et al., 2010].

Fam. Thomisidae (3)

\section{Ozyptila arctica Kulczyński, 1908}

REMARKS. Known from the Lena River Valley: TitAry \& Tas-Ary islands (Map, No. 8) [Marusik et al., 1993].

RANGE. Palaearctic-West Nearctic arcto-boreal [Nekhaeva, 2015].

\section{Xysticus albidus Grese, 1909}

MATERIAL. Zonal. Tiksi, hummocky tundra: $1 \sigma^{7}$, sifting, 20.VII.2015; lichen-moss-dwarf birch tundra: $1 \sigma^{7}$, pitfall traps, 18-20.VII.2015.

Intra. Tiksi, moss-lichen communities in rocky debris: $20^{7} O^{7}$, sifting, 20.VII.2015.

REMARKS. Known from the Lena River Valley: TitAry \& Tas-Ary islands (Map, No. 8) [Marusik et al., 1993].

RANGE. Fennoscandian-Siberian arctic [Esyunin, Efimik, 1996; Mikhailov, 2013].

\section{Xysticus canadensis Gertsch, 1934}

REMARKS. Known from the Lena River Valley: TitAry \& Tas-Ary islands (Map, No. 8) [Marusik et al., 1993].

RANGE. Holarctic boreal [Dondale, Redner, 1978; Paquin et al., 2010; Mikhailov, 2013].

\section{Discussion}

\section{Interesting findings}

Surprisingly, despite the poorness and rather high state of knowledge of the spider fauna of the Russian Arctic, it appeared still possible to find species new for science in the region. For instance, quite recently a new species, Agyneta bulavintsevi, has been described from North Yakutia (Tiksi) [Tanasevitch, 2016], and it was proved to be more widely distributed than it was suggested earlier (see the above data).

The East Siberian-Alaskan Hilaira gertschi, previously known in the Palearctic only from Wrangel Island (Chukotka Autonomous Okrug, Russia) [Eskov, 1985; Khruleva, 1987], was for the first time recorded in continental Siberia, in mountain tundras of the Kharaulakh Mountains.

Sympatric coexistence of closely related species Silometopoides pampia and S. sphagnicola in the same kind of vegetation communities (zonal tundras in Tyylaakh) was also first recorded. It was believed earlier that these species are separated territorially or biotopically [see Tanasevitch \& Rybalov, 2015].

For most species, their findings in Tiksi and/or Tyylaakh are the northernmost among known; the north border of known range appeared most advanced into the Arctic in Holminaria pallida, Silometopoides sphagnicola, Thymoites bellissimus, Tubercithorax subarcticus and Wabasso millidgei.

\section{Species distribution over kinds of plant commu- nities}

As it was said above, the analysis of species distribution over the zonal-landscape types of vegetation was based only on collecting efforts of the authors in Tyylaakh and Tiksi in 2015 (Map, No. 1 \& 2). These two local faunas located in the subzone of typical tundras of the tundra zone [Matveeva, 1998] were found to be inhabited by 45 spider species from 6 families in total. The number of species in each family and percentage in the fauna were as follows: Linyphiidae 38 species $(84.4 \%)$, Lycosidae -3 species $(6.7 \%)$, the families Dictynidae, Tetragnathidae, Theridiidae, and Thomisidae were represented by one species each (2.2\%).

Among recognized types of plant communities (zonal, intrazonal, mountainous, azonal, and anthropogenic), intrazonal communities are especially rich: 36 species. This is quite understandable because these habitats are most diverse and contain many specific microstations with less pessimal environment conditions. Araneofauna of zonal communities is almost half as poorer and contains 26 species. This relation of the species number in these two main types of plant communities of the tundra zone quite corresponds to that of, for instance, North Yamal tundras. In the latter case, araneofauna of intrazonal and zonal types includes 33 and 24 species respectively [Tanasevitch, Rybalov, 2015].

The fauna of intrazonal biotopes is not only the richest but also the most specific: 6 species, i.e., $13 \%$ of the total composition of the two local faunas have been found only there, whereas the other types of plant communities contain $1(2 \%)$ specific element each, and anthropogenously changed stations lacks them completely. Alongside, araneofauna of intrazonal communities is characterized by the minimal share of Arctic elements - 28\% (see Table 1). The percentage of purely Arctic species in the fauna probably indicates the degree of pessimal environmental conditions and is the highest in the zonal communities - $42 \%$ and the lowest in anthropogenic, 25\%. A decrease of the Arctic component share occurs due to an increase in the share of faunistic elements with more broad latitudinal dimension of the range, like in arcto-alpine, arcto-boreal and arcto-boreo-mountainous species. 
Table 1. The ratio of faunistic elements in tundra araneofaunas (Tyylaakh and Tiksi). Таблица 1. Соотношение фаунистических элементов в аранеофауне тундр (Тыылаах и Тикси).

\begin{tabular}{|l|c|c|c|c|c|}
\hline \multirow{2}{*}{$\begin{array}{c}\text { Types of vegetation } \\
\text { communities }\end{array}$} & \multirow{2}{*}{$\begin{array}{c}\text { Number } \\
\text { of } \\
\text { species }\end{array}$} & Arctic & $\begin{array}{c}\text { Faunistic elements } \\
\text { Arcto-alpine, } \\
\text { arcto-boreal, } \\
\text { arcto-boreal-montane }\end{array}$ & Boreal & P lyzonal \\
\cline { 3 - 6 } & & \multicolumn{4}{|c|}{ Number of species and their percentage in the fauna of a plant community } \\
\hline Zonal & 26 & $11(42 \%)$ & $11(42 \%)$ & 0 & $4(15 \%)$ \\
\hline Intrazonal & 36 & $10(28 \%)$ & $21(58 \%)$ & $1(3 \%)$ & $4(11 \%)$ \\
\hline Mountain & 19 & $6(32 \%)$ & $11(58 \%)$ & $1(5 \%)$ & $1(5 \%)$ \\
\hline Azonal & 18 & $7(39 \%)$ & $10(56 \%)$ & 0 & $1(6 \%)$ \\
\hline Anthropogenic & 12 & $3(25 \%)$ & $6(50 \%)$ & $1(8 \%)$ & $2(17 \%)$ \\
\hline
\end{tabular}

Boreal species are scarce in communities of local tundras: one in each of intrazonal, mountain, and anthropogenic localities, whereas in zonal and azonal communities they are completely absent. Polyzonal species are most represented in the zonal and intrazonal communities (4 species in each).

An analysis of species composition of the spider fauna of mountain tundras (studied only in Tyylaakh) has shown that this fauna is a depleted variant of the fauna of intrazonal communities rather than that of the zonal ones, and the share of purely Arctic species in the mountains $(32 \%)$ is markedly lower than in the zonal tundras (42\%). The number of spider species as well as the relation between faunistic elements are nearly the same in mountain and azonal communities, though these faunas differ considerably in composition.

There are rather few species that are specific for a certain kind of plant community. For example, polyzonal Agyneta decora was found only in the zonal tundra, and arctic Hilaira gertschi was recorded only in the mountains. Only two species live exclusively in zonal and mountain tundras: arctic Semljicola arcticus and Silometopoides pampia. Only intrazonal biotopes are inhabited by arctic Agyneta brusnewi and Alopecosa hirtipes, arcto-boreal Hilaira proletaria, typically inrazonal Bathyphantes humilis, polyzonal Dictyna major and boreal Holminaria pallida. Arctic Gibothorax tchernovi, arcto-alpine Erigone tirolensis and $\mathrm{Ha}$ lorates holmgreni avoid zonal tundras, and arcto-boreal Hilaira incondita avoids also the mountain tundras. Perhaps, zonal tundras are preferred only by arctic Mughiphantes sobrius.

Species expanding over all or almost all types of plant communities, including mounting ones, are rather numerous: arctic Alopecosa mutabilis and Diplocephalus barbiger, arcto-alpine Semljicola simplex, arctoboreal Mecynargus tundricola, Paraglyphesis polaris, Walckenaeria korobeinikovi, Pardosa septentrionalis, arcto-boreo-montane Hilaira glacialis, as well as polyzonal Pelecopsis parallela. A similar polytopic distribution in local tundras is characterstic of Erigone arctica sibirica, E. psychrophila, Hilaira nivalis, Hybauchenidium aquilonare, Masikia indistincta and Pachygnatha clercki, although these species do not penetrate into mountains.

Rocky sites stand out against intrazonal biotopes (see Figs 12-13). They are inhabited usually by a specific petrophilous fauna. For example, some species were found only in rocky debris and rocky outcrops in tundra, among and under stones, etc., i.e.: Hilaira hyperborea, Poeciloneta pallida, Proislandiana pallida, Sibirocyba incerta, Thymoites bellissimus and Holminaria pallida. There is no full confidence as regards to the latter species because of deficient information about it, but all other species from the list are typically petrophilous, and their association with these biotopes was mentioned in literature [see Marusik, 2004].

Table 2 lists all 99 species recorded in the Lena lower reaches, in the Kharaulakh Mountains, and their vicinities. Species known from the region only as based on literature data are marked by "+" and "*”. Species from the Lena valley communities (localities 8-11) are isolated into two separate columns. Of 57 species reported here, 35 (marked with an asterisk) are typically boreal - they penetrate the tundra zone but do not get out of the valley boreal communities towards the watershed, into the tundra habitats, and thus do not belong to components of the tundra fauna. This should be accounted in studying the faunal peculiarities of various natural zones and subzones. Otherwise, the real pattern of zonal faunal differentiation can be distorted due to southern faunal elements penetrating to northern and extrinsic latitudes along the river valleys.

ACKNOWLEDGEMENTS. We are greatly indebted to Yuri I. Esaulov, the Director of the Ust-Lensky Nature Reserve, for the possibility to work on the Reserve territory, as well as to Irina A. Yakshina and other members of the Reserve staff for the help in field studies. We also thank Kirill G. Mikhailov for the access to the collections of ZMMU, and also Nadezhda B. Leonova (MSU) for the help in identification of mosses and willows. The study was supported by the Russian Foundation for Basic Research, Project \#15-04-05964.

\section{References}

Chernov Yu.I. 1978. [The structure of the animal population of the Subarctic]. Moscow. Nauka Publ. 165 pp. [in Russian]. 
Chernov Yu.I., Matveeva N.V. 2002. [Landscape-zonal distribution of the arctic biota] // Uspekhi sovremennoi biologii. Vol.122. No.1. P.26-45 [in Russian].

Dondale C.D., Redner J.H., Marusik Yu.M. 1997. Spiders (Araneae) of the Yukon // Insects of the Yukon. Biological Survey of Canada (Terrestrial Arthropods). Ottawa. P.73-113.

Dondale C.D., Redner J.H. 1978. The insects and arachnids of Canada. Part 5. The crab spiders of Canada and Alaska (Araneae: Philodromidae and Thomisidae) // Research Branch Agriculture Canada Publication. Vol.1663. P.1-255.

Dondale C.D., Redner J.H. 1990. The insects and arachnids of Canada, Part 17. The wolf spiders, nurseryweb spiders, and lynx spiders of Canada and Alaska (Araneae: Lycosidae, Pisauridae, and Oxyopidae) // Research Branch Agriculture Canada Publication. Vol.1856. 383 pp.

Dondale C.D., Redner J.H., Paquin P., Levi H.W. 2003. The insects and arachnids of Canada. Part 23. The orb-weaving spiders of Canada and Alaska (Araneae: Uloboridae, Tetragnathidae, Araneidae, Theridiosomatidae). NRC Research Press, Ottawa. 371 pp.

Eskov K.Yu. 1981. [Taxonomy of spiders of the genus Hilaira (Aranei, Linyphiidae)] // Zool. Zhurn. Vol.60. No.10. P.14861493 [in Russian]

Eskov K.Yu. 1985. [Spiders of the tundra zone of the USSR] // V.I. Ovcharenko (ed.). Fauna I ekologiya paukov SSSR. Trudy Zool. Inst. Akad. nauk SSSR, Leningrad. Vol.139. P.121-128 [in Russian].

Eskov K.Yu. 1986. [On the spider genus Pero Tanasevitch, 1985 (Aranei, Linyphiidae)] // Zool. Zhurn. Vol.65. No.11. P.17381742 [in Russian].

Eskov K.Yu. 1987. [New data on the spider genus Hilaira (Aranei, Linyphiidae) in the fauna of the USSR] // Zool. Zhurn. Vol.66. No.7. P.1020-1031 [in Russian].

Eskov K.Yu. 1988. The spider genera Savignya Blackwall, Diplocephalus Bertkau and Archaraeoncus Tanasevitch (Aranei, Linyphiidae) in the fauna of Siberia and the Soviet Far East // Folia Entomol. Hung. T.49. P.13-39.

Eskov K.Yu. 1994. Catalogue of the linyphiid spiders of northern Asia (Arachnida, Araneae, Linyphiidae). Sofia-Moscow: Pensoft Publ. 144 pp.

Esyunin S.L., Efimik V.E. 1996. Catalogue of the spiders (Arachnida, Aranei) of the Urals. Moscow: KMK Scientific Press Ltd. 229 pp.

Gvozdetsky N.A., Mikhailov N.I. 1987. [Physical geography of the USSR. Asian part]. Moskva: Vysshaya Shkola Publs. 448 pp. [in Russian].

Khruleva O.A. 1987. [Invertebrates] // The flora and fauna of reserves of the USSR. Fauna of the "Wrangel Island" Reserve. Inst. Evol. Morphol. Ecol. Anim. Moscow. P.6-36 [in Russian].

Kronestedt T. 1986. Studies on species of Holarctic Pardosa groups (Araneae, Lycosidae). III. Redescriptions of Pardosa algens (Kulczyński), $P$. septentrionalis (Westring), and $P$. sodalis Holm // Entomologica Scandinavica. Vol.17. P.215-234.

Kronestedt T., Marusik Yu.M. 2011. Studies on species of Holarctic Pardosa groups (Araneae, Lycosidae). VII. The Pardosa tesquorum group // Zootaxa. Vol.3131. P.1-34.

Kulczyński V. 1908. Araneae et Oribatidae expeditionum Rossicarum in insulas Novo-Sibiricas annis $1885-1886$ et 1900 1903 susceptarum // Mém. Acad. Imp. Sci. St.-Petersbourg. Sér.VIII. Cl. phys.-math. Vol.18. No.7. P.I-IV, 1-97, P1.I-III.

Logunov D.V. 1996. A critical review of the spider genera Apollophanes O.P.-Cambridge, 1898 and Thanatus C.L. Koch, 1837 in North Asia (Araneae, Philodromidae) // Revue Arachnologique. T.11. Fasc.13. P.133-202.

Marusik Yu.I., Fritzén N.R. 2011. On a new Dictyna species (Araneae, Dictynidae) from the northern Palaearctic confused with the East Siberian D. schmidti Kulczyński, 1926 // ZooKeys. Vol.138. P.93-108.

Marusik Yu.I., Omelko M.M., Koponen S. 2011. On the distribution of Gnaphosa orites (Araneae: Gnaphosidae) with a note on its southernmost record, from the Russian Far East // Acta Arachnologica. Vol.60. No.1. P.51-53.
Marusik Yu.M. 2004. Fauna and populations of the petrophilous spiders (Arachnida: Araneae) of north-east Siberia and northwest Canada // D.V. Logunov, D. Penney (eds.). European Arachnology 2003. Proc. $21^{\text {st }}$ Europ. Colloq. Arachnol., St.Petersb., 4-9 Aug. 2003. Moscow. KMK Sci. Press Ltd. P.185200.

Marusik Yu.M., Azarkina G.N., Koponen S. 2003. A survey of East Palaearctic Lycosidae (Aranei). II. Genus Acantholycosa F. Dahl, 1908 and related new genera // Arthropoda Selecta. Vol.12. No.2. P.101-148.

Marusik Yu.M., Böcher J., Koponen S. 2006. The collection of Greenland Spiders (Aranei) kept in the Zoological Museum, University of Copenhagen // Arthropoda Selecta. Vol.15. No.1. P.59-80.

Marusik Yu.M., Eskov K.Yu., Koponen S., Vinokurov N.N. 1993. A check-list of the spiders (Aranei) of Yakutia, Siberia // Arthropoda Selecta. Vol.2. No.2. P.63-79.

Marusik Yu.M., Gnelitsa V.A., Koponen S. 2008. A survey of Holarctic Linyphiidae (Araneae). 3. A review of the genus Praestigia Millidge, 1954 // Bull. Brit. arachnol. Soc. Vol.14. Pt.5. P.213-231.

Marusik Yu.M., Koponen S. 2001. Description of a new species and new records of some species of the genus Gnaphosa (Araneae: Gnaphosidae) from the east Palaearctic // Acta Arachnologica. Vol.50. No.2. P.135-144.

Marusik Yu.M., Logunov D.V., Koponen S. 2000. Spiders of Tuva, South Siberia. Magadan: IBPN FEB RAS. 252 pp.

Matveeva N.V. 1998. [Zonation of Arctic Vegetation] // Trudy Botanicheskogo Instituta im. V.L. Komarova Ross. Akad. Nauk. St.-Petersburg: Nauka Publ. 200 pp.

Mikhailov K.G. 2013. The spiders (Arachnida: Aranei) of Russia and adjacent countries: a non-annotated checklist // Arthropoda Selecta. Suppl. No.3. P.1-264.

Nekhaeva A.A. 2015. An annotated check-list of spiders (Arachnida: Aranei) of the Khibiny Mountains, Kola Peninsula, Russia // Arthropoda Selecta. Vol.24. No.4. P.451-472.

Omelko M.M., Marusik Yu.M. 2013. A survey of East Palaearctic Lycosidae (Araneae). 9. A review of Sibirocosa with a description of three new species // Zootaxa. Vol.3666. No.3. P.319-330.

Paquin P., Buckle D.J., Duperre N., Dondale C.D. 2010. Checklist of the spiders (Araneae) of Canada and Alaska // Zootaxa. Vol.2461. P.1-170.

Platnick N.I., Dondale C.D. 1992. The insects and arachnids of Canada. Part 19. The ground spiders of Canada and Alaska (Araneae: Gnaphosidae) // Research Branch Agriculture Canada Publication. Vol.1875. 297 pp.

Rybalov L.B. 2002. [Zonal-landscape change in population of soil invertebrates in Near-Yenissei region of Middle Siberia and the role of temperature adaptations in meridional (zonal) distribution of invertebrates // Russian Entomological Journal. Vol.11. No.1. P.77-86 [in Russian].

Slowik J., Sikes D.S. 2013. Synonymy of four Pardosa species (Araneae: Lycosidae) undiagnosable without geography // The Journal of Arachnology. Vol.41. P.327-334.

Szita É., Logunov D. 2008. A review of the Histrio group of the spider genus Philodromus Walckenaer, 1826 (Araneae, Philodromidae) of the Eastern Palaearctic Region // Acta Zoologica Academiae Scientiarum Hungaricae. Vol.54. No.1. P.23-73.

Tanasevitch A.V., Kamayev I.O. 2011. [Spiders of Kola Peninsula, Russia (Arachnida: Aranei)] // Caucasian Entomological Bulletin. Vol.7. No.1. P.7-32 [in Russian].

Tanasevitch A.V., Koponen S. 2007. Spiders of the southern tundra in the Russian Plain // Arthropoda Selecta. Vol.15. No.4. P.295-345.

Tanasevitch A.V., Rybalov L.B. 2010. On spiders from tundra zone of Kola Peninsula, Russia (Arachnida, Aranei) // Arthropoda Selecta. Vol.19. No.1. P.41-56.

Tanasevitch A.V., Rybalov L.B. 2015. Fauna and distribution of spiders (Aranei) of the arctic tundra in northern Yamal Peninsula, Russia // Arthropoda Selecta. Vol.24. No.2. P.215-230.

Responsible editor K.G. Mikhailov 


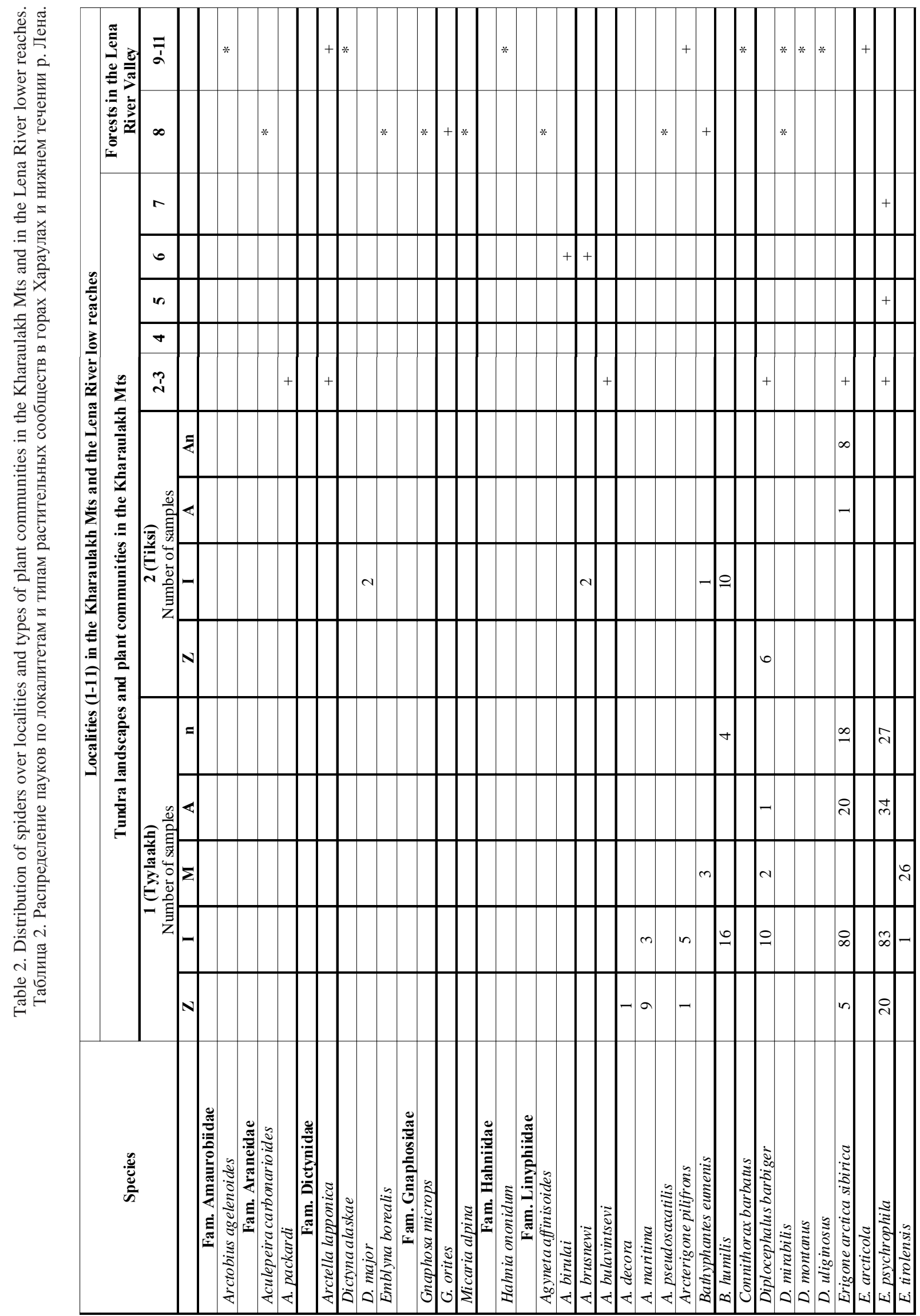




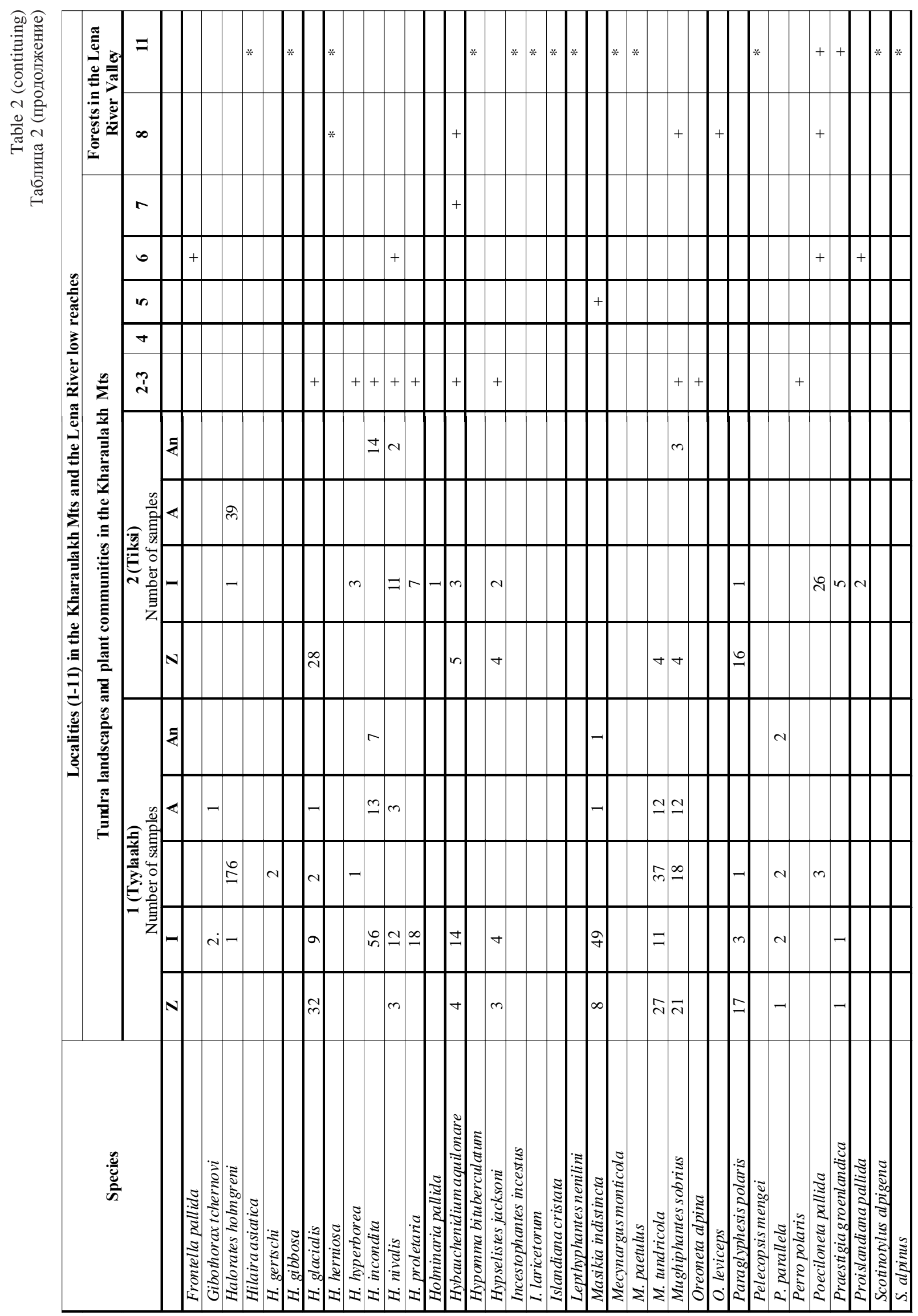




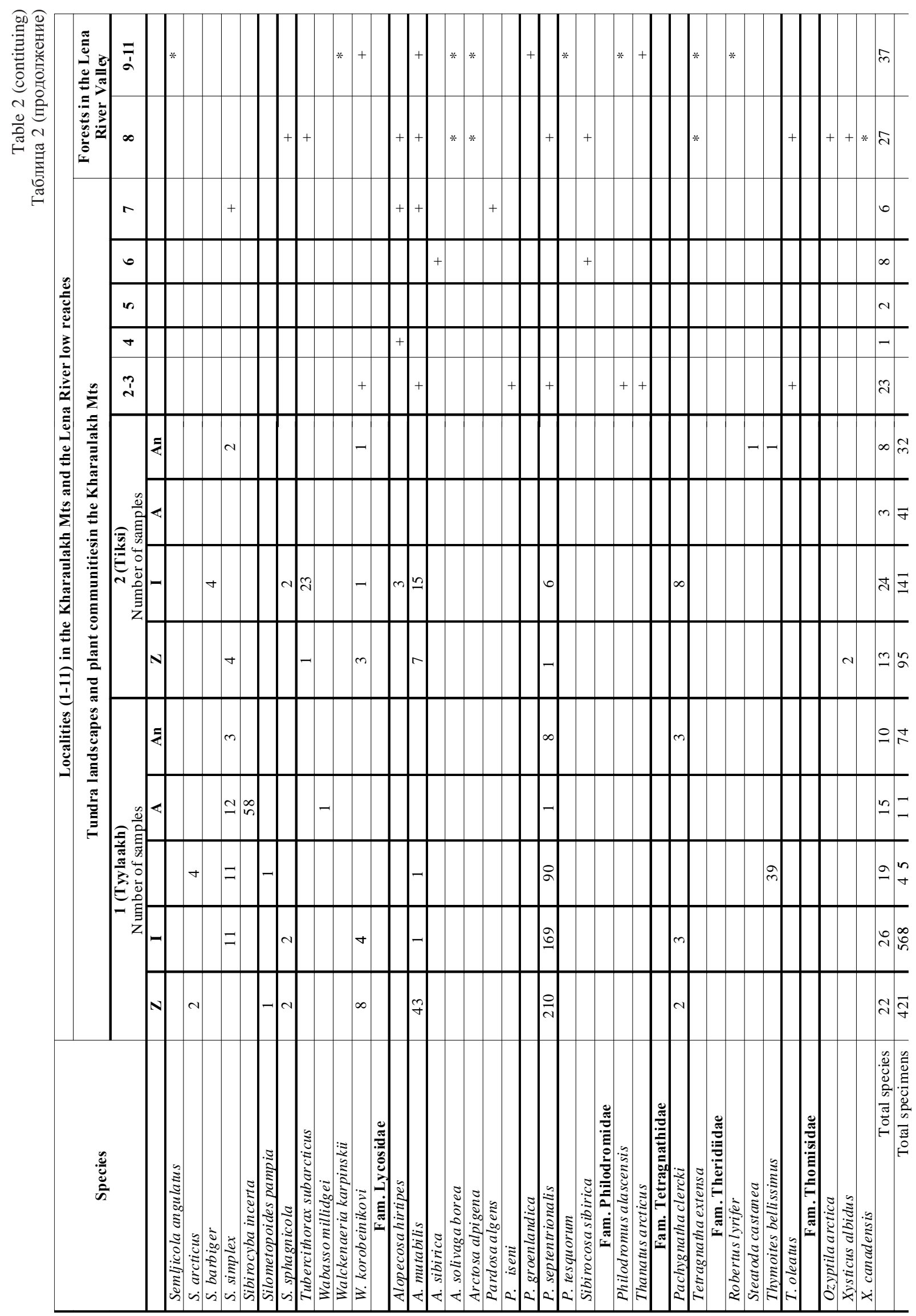

